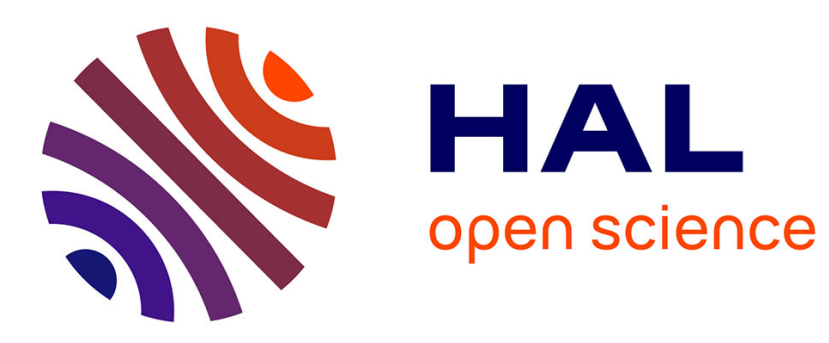

\title{
Adapted generalized lifting schemes for scalable lossless image coding
}

Hocine Bekkouche, Michel Barret, Jacques Oksman

\section{To cite this version:}

Hocine Bekkouche, Michel Barret, Jacques Oksman. Adapted generalized lifting schemes for scalable lossless image coding. Signal Processing, 2008, Vol. 88 ((11)), pp.2790-2808. 10.1016/j.sigpro.2008.06.003 . hal-00287898

\section{HAL Id: hal-00287898 \\ https://hal-centralesupelec.archives-ouvertes.fr/hal-00287898}

Submitted on 13 Jun 2008

HAL is a multi-disciplinary open access archive for the deposit and dissemination of scientific research documents, whether they are published or not. The documents may come from teaching and research institutions in France or abroad, or from public or private research centers.
L'archive ouverte pluridisciplinaire HAL, est destinée au dépôt et à la diffusion de documents scientifiques de niveau recherche, publiés ou non, émanant des établissements d'enseignement et de recherche français ou étrangers, des laboratoires publics ou privés. 
Adapted generalized lifting schemes for scalable lossless image coding

Hocine Bekkouche ${ }^{1 \star}$, Michel Barret ${ }^{1}$ and Jacques Oksman ${ }^{2}$

${ }^{1}$ Information, Multimodality and Signal Team, SUPELEC, 2 rue É. Belin, 57070 Metz, France.

2 Signals and Electronic Systems Team, SUPELEC,

Plateau de Moulon, 91192 Gif-sur-Yvette, France.

FirstName.Name@supelec.fr

${ }^{\star}$ This work was partially supported by the Lorraine Region.

June 2, 2008 


\begin{abstract}
Still image coding occasionally uses linear predictive coding together with multi-resolution decompositions, as may be found in several papers. Those related approaches do not take into account all the information available at the decoder in the prediction stage. In this paper, we introduce an adapted generalized lifting scheme in which the predictor is built upon two filters, leading to taking advantage of all this available information. With this structure included in a multi-resolution decomposition framework, we study two kinds of adaptation based on least squares estimation, according to different assumptions, which are either a global or a local second order stationarity of the image. The efficiency in lossless coding of these decompositions is shown on synthetic images and their performances are compared with those of well-known codecs (S+P, JPEG-LS, JPEG2000, CALIC) on actual images. Four images' families are distinguished: natural, MRI medical, satellite and textures associated with fingerprints. On natural and medical images, the performances of our codecs do not exceed those of classical codecs. Now for satellite images and textures, they present a slightly noticeable (about 0.05 to $0.08 \mathrm{bpp}$ ) coding gain compared to the others that permit a progressive coding in resolution, but with a greater coding time.
\end{abstract}

Keywords: Wavelets, Adapted filter banks, Adapted lifting scheme, Adaptive filtering, Lossless image compression, Still image compression, Progressive coding, Multiresolution analysis.

\title{
1 Introduction
}

The lossless image compression finds applications in satellite and medical image processing, where a lossy or near lossless coding is not satisfactory. However, in many applications of lossless coding, from time to time, lossless at full resolution is not possible because the transmission channel has a limited bandpass and then coding with a smaller resolution is better than no transmission at all. In other applications, customers need lossless coding at full resolution and other ones are satisfied with smaller resolutions of the same images. Therefore, embedded progressive coding from low resolution to lossless full resolution can be a good compromise in many applications. This coding allows to reconstruct from a truncated bit flow a decompressed image, which has a smaller resolution than the encoded one. As and when the data are received, the user is capable of enhancing the image resolution, until it reaches the original quality and resolution. 
It is well known that bi-orthogonal wavelet decompositions are efficient for lossy and near lossless image compression [1], this is why they are used in the ISO JPEG2000 standard. The lifting scheme, introduced by Sweldens [2] in order to construct wavelet decompositions by a simple, reversible and fast process, found quickly its main application in lossless image compression. In this case, a nonlinear filter bank with critical sampling and perfect reconstruction is obtained, with nonlinearities which are limited to truncations (i.e., rounding to the nearest integer) [3]. Moreover, Daubechies and Sweldens showed that any bi-orthogonal wavelet decomposition with FIR (Finite Impulse Response) filters can be represented by a lifting scheme [4] and, therefore, all the well-known wavelets used in lossy image codecs can be quite closely approximated by integer-to-integer wavelets. The performances in lossy and lossless image compression of integer-to-integer wavelets and the S+P transform by Said and Pearlman [5] are evaluated in [6]. Hampson and Pesquet [7] proposed a structure which is more general than the lifting scheme, with an arbitrary number of channels and arbitrary nonlinear filters. It is interesting to note the simplicity of this structure and the way the perfect reconstruction is performed in an inherent manner by a synthesis filter bank "mirror" of the analysis filter bank, as in the lifting scheme. That structure, with nonlinear prediction filters based on image segmentations, has been applied for still image and video coding by Amonou and Duhamel [8].

In the standard wavelet decompositions, the filter coefficients are fixed: they do not adapt to the image as best possible. However, the lifting scheme gives an interpretation in terms of estimation (or prediction) of perfect reconstruction filter banks, associated with multiresolution decompositions. Now, linear prediction coding (LPC) proved its great efficiency for speech coding; it found applications in mobile telephones. Therefore it is natural to study LPC in image coding. About fifteen years ago, adaptive linear predictions using least squares estimation (LSE) algorithms were tested for image compression (see [9] and its bibliography, or later [10]), but they were not associated with dyadic decompositions and consequently they were not suitable for progressive coding. More recently, Gerek and Çetin [11] used the lifting scheme with adaptive predict steps: the filter coefficients were updated to each pixel of the image, thanks to a conventional stochastic gradient algorithm, in order to minimize the variance of the detail signal. In [12], Boulgouris, Tzovaras and Strintzis expressed each filter of the optimal $M$-subband analysis filter bank as a function of the power spectral density (PSD) of the input image. They assumed the entire image is a wide sense stationary (WSS) 
signal. The optimum is achieved by minimizing the mean squared error of prediction for each of the $M-1$ detail signals. Two kinds of parameterized models were assumed for the PSD of the image, i.e., the adaptation is optimum only if the PSD of the image belongs to a set of two models. The filters of the update steps did not adapt to the image, they were identical with those encountered in the lifting scheme of well-known wavelets. To improve the prediction whenever the global WSS assumption is invalid, the linear predictors were enhanced by nonlinear means, namely by directional post-processing in the quincunx decimation case, and by adaptive-length post-processing in the separable (row-column) decimation case. In [13], the authors chose locally, among a finite dictionary of wavelet filters, the filter that must be applied to the current pixel depending on its proximity with an outline: the closer the pixel is to an outline, the smaller the impulse response support of the analysis filter. In [14], the authors studied the optimization of a lifting scheme (for both the predict and update steps) associated with 2-fold quincunx decimation. They imposed constraints to the filters in order to avoid overflow and they applied their filter banks to lossy image compression.

In each of the above mentioned papers with adapted prediction filters, we can notice that all the information available at the decoder is not taken into account in the "predict" step" ${ }^{1}$ Indeed, after the 2-fold decimation, the pixels of a subband, say $x_{2}$, are predicted as a linear combination of the pixels of the other subband, say $x_{1}$, and the pixels of subband $x_{2}$ are not involved in the observation vector, whereas they could be! As is done in the classical LPC. In [15], we introduced an adapted integer-to-integer multiresolution decomposition, based on LSE and assuming global second order stationarity of the image, which takes advantage of all the information available at the decoder, and we applied it to lossless image coding. In [16], we completed this decomposition by introducing another adaptation, which assumes only local stationarity in the image. The reason that led us to carry out this study lies in the fact that the image models are not fully appropriate for entire images, they are better justified for well-chosen parts of the images taken separately. Those parts are the textured regions that can be found in most kinds of images. Then, in [17] we compared the performances of these decompositions in lossless coding of satellite and medical MRI images with well-known codecs.

In this paper, we complete the results of the conference papers [15], [16] and [17] and provide more details

\footnotetext{
${ }^{1}$ We should say "estimation" step, since it is not a prediction problem, but an estimation problem in estimation theory; nevertheless we chose the vocabulary used in filter bank theory.
} 
and full proofs. First, we present the adapted generalized lifting scheme framework, which is shared both by locally and globally adapted estimation methods - we shall call them respectively LAE and GAE below. In Sections 3.1 and 3.2, the GAE and LAE methods are explained in details. Their efficiency in lossless coding is shown on synthetic images (Section 4) and their performances are compared with those of well-known codecs (S+P [5, 18], LOCO I [19, 20], CALIC [21, 22] and Jasper [23]) on actual images (Section 5). We considered four families of images (natural, medical MRI, satellite and textures with fingerprints).

In the following, $\mathbb{Z}$ denotes the set of all integers. For a matrix $\mathbf{A}, \mathbf{A}^{T}$ denotes its transpose. Underlined lower case letters denote vectors, which are identified with the column matrix of their coordinates. The symbol E denotes the mathematical expectation.

\section{$2 \quad$ Adapted generalized lifting schemes}

In this section we begin by presenting a short overview of the generalized lifting scheme in the monodimensional (1-D) case, then we extend it to the 2-D case, clarifying the integer-to-integer variant and the adaptation of the filters. Furthermore we explain how the generalized lifting scheme can be used in a multiresolution framework, permitting a progressive coding in resolution, and we specify what kind of extension is applied to the edges of the image during the filtering. As introduction, we recall in Fig. 1 the principle of the standard lifting scheme (without adaptation) for a perfect reconstruction filter bank with two subbands, $L$ "Predict" steps (denoted $\left.P_{1}(z), P_{2}(z), \ldots, P_{L}(z)\right)$ and $L$ "Update" steps (denoted $U_{1}(z), U_{2}(z), \ldots, U_{L}(z)$ ). The synthesis filter is easily obtained from the analysis filter by a simple mirror symmetry.

\subsection{Principle of the method in the 1-D case}

The general diagram is presented in Fig. 2. The polyphase components of the input signal are obtained by a polyphase decomposition (i.e., a lazy wavelet transform) $[24,25]$. One of the components, $x_{2}(n)$, is estimated with the use of two filters $A(z)$ and $B(z)$, the signal of details corresponds to the error of estimation $x_{2}(n)-\hat{x}_{2}(n)$. The approximate signal $x_{\ell}$ is just the polyphase component $x_{1}$ of the input signal $x$. This is a generalized version of the standard lifting scheme (see Fig. 1), since the filter $B(z)$ is added in order to 
improve the prediction. The polyphase matrix of the analysis filter bank is equal to

$$
\mathbf{R}(z)=\left[\begin{array}{cc}
1 & 0 \\
-A(z) & 1-B(z)
\end{array}\right] .
$$

and then, for perfect reconstruction, the polyphase matrix of the synthesis filter bank is equal to

$$
\mathbf{R}^{-1}(z)=\frac{1}{1-B(z)}\left[\begin{array}{cc}
1-B(z) & 0 \\
A(z) & 1
\end{array}\right]
$$

In order to reconstruct the input signal perfectly with the synthesis filter bank, it is clear that the filter $B(z)$ must be causal, since only the passed samples of $x_{2}(n)$ are available at the decoder for the reconstruction of $\hat{x}_{2}(n)$. Nevertheless, the causality of the filter $A(z)$ is not required, since all the samples of the polyphase component $x_{1}$ are available as inputs of the synthesis filter bank. Another major condition for perfect reconstruction is the BIBO (bounded-input-bounded-output) stability of the filter $\frac{1}{1-B(z)}$. Indeed, small perturbations, like round-off errors, would lead to a divergence of the reconstruction algorithm when this filter is not stable. Therefore a stability test for 1-D filter [26] is required in the case of linear filter banks.

The adaptation of the filters and the properties of the generalized lifting scheme are very similar in the 1-D and 2-D cases, so in order to be brief, we present them only in the 2-D case.

\subsection{Generalized lifting scheme for 2-D signals}

The extension to the 2-D case of the previous generalized lifting scheme can be carried out in two different ways, according to the polyphase decomposition which can be either a 2-fold quincunx decimation or a 2 -fold separable (row / column) decimation. In both cases, both of the 2-D filters $A\left(z_{1}, z_{2}\right)$ and $B\left(z_{1}, z_{2}\right)$ are not separable a priori. As in the 1 -D case, the causality or semi-causality of the filter $B\left(z_{1}, z_{2}\right)$ is required for perfect reconstruction. Moreover, the 2-D stability test [26] of the filter $\frac{1}{1-B\left(z_{1}, z_{2}\right)}$ is also required in the linear case. We shall see in Subsection 2.4 (Remark 2) that the stability test is not required for the integer-to-integer generalized lifting scheme.

The filters in Fig. 3 are expressed in terms of their transfer functions

$$
A\left(z_{1}, z_{2}\right)=\sum_{(i, j) \in \Delta_{1}} a_{i j} z_{1}^{-i} z_{2}^{-j} \quad \text { and } \quad B\left(z_{1}, z_{2}\right)=\sum_{(i, j) \in \Delta_{2}} b_{i j} z_{1}^{-i} z_{2}^{-j}
$$


where $\Delta_{1}$ is any subset of $\mathbb{Z}^{2}$ (no condition of causality is imposed) and $\Delta_{2}$ is a subset of a non-symmetrical half plane (NSHP) of $\mathbb{Z}^{2}$ to ensure the semi-causality of $B\left(z_{1}, z_{2}\right)$.

The prediction of $x_{2}(m, n)$ is given by the equation

$$
\hat{x}_{2}(m, n)=\sum_{(i, j) \in \Delta_{1}} a_{i j} x_{1}(m-i, n-j)+\sum_{(i, j) \in \Delta_{2}} b_{i j} x_{2}(m-i, n-j)
$$

and the criterion used for the filters coefficients adaptation is the minimization of the mean squared error $\mathrm{E}\left[\left|x_{2}(m, n)-\hat{x}_{2}(m, n)\right|^{2}\right]$

\subsection{Adaptation of the filters}

To solve the problem of 2-D LSE (4), we first scan the elements of sub-sets $\Delta_{1}$ and $\Delta_{2}$ in a preset order. We build thus, from the respective families of pixels $\left.x_{1}(m-i, n-j)\right|_{(i, j) \in \Delta_{1}}$ and $\left.x_{2}(m-i, n-j)\right|_{(i, j) \in \Delta_{2}}$, observation vectors $\underline{y}_{1}(m, n)$ and $\underline{y}_{2}(m, n)$ of respective sizes $r_{1}$ and $r_{2}$. The scans chosen for the description of $\Delta_{1}$ and $\Delta_{2}$ impose an organization of the coefficients $\left.a_{i, j}\right|_{(i, j) \in \Delta_{1}}$ and $\left.b_{i j}\right|_{(i, j) \in \Delta_{2}}$ of filters $A\left(z_{1}, z_{2}\right)$ and $B\left(z_{1}, z_{2}\right)$ as elements of the respective vectors $\underline{a}$ and $\underline{b}$ so that

$$
\hat{x}_{2}(m, n)=\left[\underline{a}^{T}, \underline{b}^{T}\right]\left[\begin{array}{l}
\underline{y}_{1}(m, n) \\
\underline{y}_{2}(m, n)
\end{array}\right]=\underline{c}^{T} \underline{y}(m, n)
$$

with $\underline{c}^{T}=\left[\underline{a}^{T}, \underline{b}^{T}\right]$ and $\underline{y}(m, n)^{T}=\left[\underline{y}_{1}(m, n)^{T}, \underline{y}_{2}(m, n)^{T}\right]$.

While applying, for example the principle of orthogonality, one finds that vector $\underline{c}$, of dimension $r=r_{1}+r_{2}$, is the solution of the Yule-Walker equations

$$
\Gamma_{Y} \underline{c}=\underline{\gamma}_{y x}
$$

with $\Gamma_{Y}=\mathrm{E}\left[\underline{y}(m, n) \underline{y}(m, n)^{T}\right]$ and $\underline{\gamma}_{y x}=\mathrm{E}\left[\underline{y}(m, n) x_{2}(m, n)\right]$. We shall see thereafter how to estimate these mathematical expectations from the data. We shall distinguish two cases, according to the assumption of wide sense stationarity made on the signal $x(m, n)$ : global (i.e., on the entire image) in Section 3.1 or local in Section 3.2.

Remark 1 For a WSS signal $x(m, n)$, it is well known in estimation theory that the residue of the linear least squares estimation, which corresponds to the image of details

$$
x_{h}(m, n)=x_{2}(m, n)-\hat{x}_{2}(m, n),
$$


is uncorrelated with the observations $\left.x_{1}(m-i, n-j)\right|_{(i, j) \in \Delta_{1}}$ and $\left.x_{2}(m-i, n-j)\right|_{(i, j) \in \Delta_{2}}$. Moreover, by extending well known results on linear least squares estimation to the 2-D case, it is clear that if signal $x(m, n)$ is WSS and if the supports $\Delta_{1}$ and $\Delta_{2}$ tend toward infinite ones (i.e., $\Delta_{1}=\mathbb{Z}^{2}$ and $\Delta_{2}$ is a NSHP), then the residue $x_{h}(m, n)$ tends toward a white noise. That explains why, when the supports are large enough, the coefficients close to $x_{h}(m, n)$ are very slightly correlated between them and with the coefficients of subband $x_{\ell}$. This fact is valid even when the assumption of second order stationarity is just locally satisfied.

\subsection{Integer-to-integer generalized lifting scheme}

When implemented with a fixed (or a floating) point arithmetic processor, the reversibility of the decomposition is not ensured with equation (7), since the filters coefficients are not integers. To avoid this problem, it is enough to round the estimation to the nearest integer before removing it from the exact value in order to generate the detail signal, which becomes

$$
x_{h}(m, n)=x_{2}(m, n)-\left\lfloor\sum_{(i, j) \in \Delta_{1}} a_{i, j} x_{1}(m-i, n-j)+\sum_{(i, j) \in \Delta_{2}} b_{i, j} x_{2}(m-i, n-j)+\frac{1}{2}\right\rfloor,
$$

where $\lfloor x\rfloor$ denotes the largest integer not greater than $x$. Thus, we obtain the integer-to-integer generalized lifting scheme.

Remark 2 It is important to notice here that if the coder and the decoder use the same floating point arithmetic (with the same round off rule) to calculate the expression between $\lfloor$ and $\rfloor$ in the equation (8), then the decomposition is perfectly reversible, since $x_{h}(m, n)$ and $x_{2}(m, n)$ are integers and arithmetic operations between integers are perfectly reversible (when no overflow is detected) on any processor. Moreover, this reasoning shows that the perfect reversibility remains valid even when filter $\frac{1}{1-B\left(z_{1}, z_{2}\right)}$ is unstable. Therefore the BIBO stability test is not required, for the integer-to-integer generalized lifting scheme to ensure perfect reconstruction.

\subsection{Multi-resolution decomposition}

The multi-resolution decomposition of a 2-D signal is based on the decomposition shown in Fig. 3. We first explain one level of decomposition in four subbands: the input signal $x(m, n)$ is divided into two subband signals $x_{1}$ and $x_{2}$ by a polyphase decomposition; the signal of approximation $x_{\ell}$ is then equal 
to $x_{1}$ and the signal of details $x_{h}$ is obtained according to the relation (7) (or (8) for integer-to-integer decomposition). The same process is applied to $x_{\ell}$ in order to generate the two signals $x_{\ell \ell}$ and $x_{\ell h}$ of subbands LL and LH (of course, if the 2-fold decimation is separable then the polyphase decomposition is alternatively applied to rows and columns). However, contrary to what is usually done - in particular in the case of dyadic wavelet decomposition - , to generate the two signals $x_{h \ell}$ and $x_{h h}$ of subbands HL and $\mathrm{HH}$, only a polyphase decomposition is applied to signal $x_{h}$, as indicated on Fig. 4 . Indeed, after obtaining the signal $x_{h}$ according to the process in Fig. 3, the correlation between adjoining samples of $x_{h}$ almost vanishes (see Remark 1). Consequently, the reduction in variance obtained by applying a predict step after the polyphase decomposition to generate $x_{h h}$ is so weak that we preferred not to use it, thus reducing the complexity of the codec. In order to have a decomposition on several levels, the same process is applied recursively on the signal of approximation $x_{\ell \ell}$.

\subsection{Optional update and predict steps with fixed coefficients filters}

In order to allow a progressive coding in resolution, it is necessary to avoid the artefacts (spectral aliasing) due to the 2-fold decimation when the resolution becomes low. For this we recommend to apply some update and predict steps with fixed coefficient filters corresponding to well known wavelet decomposition in the adapted generalized lifting scheme, between the polyphase decomposition and the adapted predict step. In our experiments, we chose the $S$-transformation, because of its low complexity; it consists in the two steps $($ for mono-dimensional signal $x(n)): x_{h}(n)=x(2 n)-x(2 n+1)$ and $x_{\ell}(n)=x(2 n+1)+\left\lfloor\frac{x_{h}(n)}{2}\right\rfloor$.

\subsection{Symmetrical or zero-padding extension of the image}

An image is a 2-D signal with finite support, therefore a strategy must be adopted to filter near the edges of the image. Generally, the strategy consists in either zero padding, or a periodical extension, or a symmetrical extension. In our tests, we chose the last one when the decimation is separable, since it avoids discontinuities and thus generally gives better performances in coding. Nevertheless, with quincunx decimation, we adopted zero padding to simplify the implementation of the codec. 


\section{Two approaches of Least Squares Estimation}

We distinguish two kinds of adaptation for the filters $A\left(z_{1}, z_{2}\right)$ and $B\left(z_{1}, z_{2}\right)$ according to the assumptions of global or local wide sense stationarity of the encoded image.

\subsection{Globally adapted estimation (GAE) method}

\subsubsection{Choice of the criterion}

The input signal is supposed to be a realization of a 2-D WSS stochastic process. The sub-set $\Delta_{1} \subset \mathbb{Z}^{2}$ is a rectangle centered in $(0,0): \Delta_{1}=\Delta_{1}^{\prime}$ with

$$
\Delta_{1}^{\prime}=\left\{(i, j) \in \mathbb{Z}^{2}:|i| \leq p \text { and }|j| \leq q\right\}
$$

and the sub-set $\Delta_{2} \subset \mathbb{Z}^{2}$ is a bounded NSHP: $\Delta_{2}=\Delta_{2}^{\prime}$ with

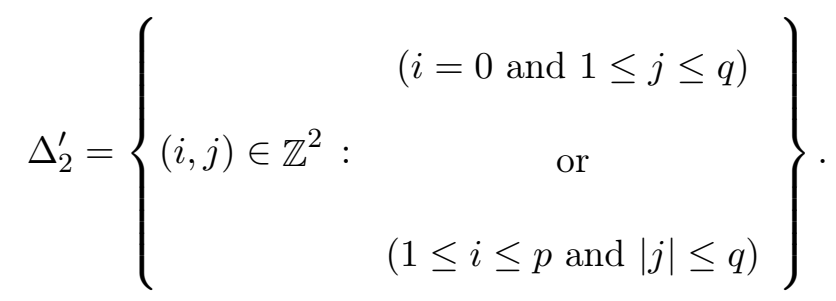

The vectors, $\underline{y}_{1}(m, n)=\left[x_{1}(m+p, n+q), \ldots, x_{1}(m+p, n-q), x_{1}(m+p-1, n+q), \ldots, x_{1}(m+p-1, n-q)\right.$, $\left.\ldots, x_{1}(m-p+1, n+q), \ldots, x_{1}(m-p+1, n-q), x_{1}(m-p, n+q), \ldots, x_{1}(m-p, n-q)\right]^{T}$ of dimension $r_{1}=(2 p+1)(2 q+1)$ and $\underline{y}_{2}(m, n)=\left[x_{2}(m, n-1), \ldots, x_{2}(m, n-q), x_{2}(m-1, n+q), \ldots, x_{2}(m-1, n-q)\right.$, $\left.\ldots, x_{2}(m-p+1, n+q), \ldots, x_{2}(m-p+1, n-q), x_{2}(m-p, n+q), \ldots, x_{2}(m-p, n-q)\right]^{T}$ of dimension $r_{2}=$ $p(2 q+1)+q$, contain the values of the respective samples $\left.x_{1}(m-i, n-j)\right|_{(i, j) \in \Delta_{1}}$ and $\left.x_{2}(m-i, n-j)\right|_{(i, j) \in \Delta_{2}}$. The vectors $\underline{a}$ and $\underline{b}$ can be expressed as $\underline{a}=\left[a_{-p,-q}, \ldots, a_{-p, q}, a_{-p+1,-q}, \ldots, a_{-p+1, q}, \ldots, a_{p,-q}, \ldots, a_{p, q}\right]^{T}$ and $\underline{b}=\left[b_{0,1}, \ldots, b_{0, q}, b_{1,-q}, \ldots, b_{1, q}, \ldots, b_{p,-q}, \ldots, b_{p, q}\right]^{T}$.

After the polyphase decomposition applied to the image $x(m, n)$ of dimension $M \times N$, the images of the subbands $x_{1}(m, n)$ and $x_{2}(m, n)$ have the respective dimensions $M_{1} \times N_{1}$ and $M_{2} \times N_{2}$, the support of $x_{2}(m, n)$ is then $0 \leq m<M_{2}$ and $0 \leq n<N_{2}$. According to the orders of the filters $A\left(z_{1}, z_{2}\right)$ and $B\left(z_{1}, z_{2}\right)$, the vectors $\underline{y}_{1}(m, n)$ and $\underline{y}_{2}(m, n)$ can have supports which are located more or less beside the horizon of observation of $x$, hence in order to compute the actual matrices $\Gamma_{Y}$ and $\underline{\gamma}_{y x}$ of the relation (6), the observations must be extended beside the horizon of observation. Three methods have been tested. 
Pre-windowed method. The subband signals $x_{1}(m, n)$ and $x_{2}(m, n)$ are supposed to be null for $m<0$ or $n<0$ and no hypothesis is made on their values for $m \geq M_{2}$ or $n \geq N_{2}$.

Autocorrelation method. The subband signals $x_{1}(m, n)$ and $x_{2}(m, n)$ are supposed to be null beside the horizon of observation, i.e., for $m<0$ or $n<0$ and for $m \geq M_{2}$ or $n \geq N_{2}$.

Covariance method. No hypothesis is made on the values of $x_{1}$ and $x_{2}$ beside the horizon of observation.

Our simulations on actual images show that the autocorrelation method gives quite often and on average first order entropies slightly greater than each of the two other methods. In the continuation of our simulations, we use the pre-windowed method, which gives often and on average performances similar to the covariance method with a weaker complexity ${ }^{2}$.

The criterion to be minimized is thus given by

$$
J_{1}=\frac{1}{\left(M_{2}-p\right)\left(N_{2}-q\right)} \sum_{m=0}^{M_{2}-p-1} \sum_{n=0}^{N_{2}-q-1} x_{h}(m, n)^{2} \simeq \mathrm{E}\left[x_{h}(m, n)^{2}\right]
$$

Let us gather the elements $\left.x_{h}(m, n)\right|_{0 \leq m<M_{2}-p, 0 \leq n<N_{2}-q}$ in the vector $\underline{\mathcal{X}}_{h}$ by scanning $x_{h}$ row after row: $\underline{\mathcal{X}}_{h}=\left[x_{h}(0,0) \cdots x_{h}\left(0, N_{2}-q-1\right) \cdots x_{h}\left(M_{2}-p-1,0\right) \cdots x_{h}\left(M_{2}-p-1, N_{2}-q-1\right)\right]^{T}$ we then get the relation $\underline{\mathcal{X}}_{h}=\underline{\mathcal{X}}_{2}-\mathcal{Y} \underline{\underline{c}}$, with $\underline{\mathcal{X}}_{2}=\left[x_{2}(0,0) \cdots x_{2}\left(0, N_{2}-q-1\right) \cdots x_{2}\left(M_{2}-p-1,0\right) \cdots x_{2}\left(M_{2}-p-1, N_{2}-q-1\right)\right]^{T}$ and the matrix of dimension $\left(M_{2}-p\right)\left(N_{2}-q\right) \times r$, which is equal to $\mathcal{Y}=\left[\underline{y}(0,0) \cdots \underline{y}\left(0, N_{2}-q-1\right) \cdots \underline{y}\left(M_{2}-p-\right.\right.$ $\left.1,0) \cdots \underline{y}\left(M_{2}-p-1, N_{2}-q-1\right)\right]^{T}$. The criterion (11) to be minimized can be written $\left(M_{2}-p\right)\left(N_{2}-q\right) J_{1}=$ $\left\|\underline{\mathcal{X}}_{h}\right\|_{2}^{2}=\left\|\underline{\mathcal{X}}_{2}-\mathcal{Y} \underline{\underline{c}}\right\|_{2}^{2}=\left[\underline{\mathcal{X}}_{2}-\mathcal{Y} \underline{\underline{c}}\right]^{T}\left[\underline{\mathcal{X}}_{2}-\mathcal{Y} \underline{c}\right]$ and the vector $\underline{\hat{c}}$ is the solution of the Yule-Walker equations

$$
\mathcal{Y}^{T} \mathcal{Y} \underline{\hat{c}}=\mathcal{Y}^{T} \underline{\mathcal{X}}_{2}
$$

\subsubsection{Complexity of the method}

In the GAE method, the stage that requires most of the arithmetic operations is the calculation of the matrix $\mathcal{Y}^{T} \mathcal{Y}$ of the Yule-Walker equations. Therefore, it is important to reduce its complexity by using the great redundancy that exists between its elements. The fast computation of covariance matrix associated to 2-D

\footnotetext{
${ }^{2}$ We emphasize that the three methods of extension at the edges of the image above mentioned are useful only for clarifying the matrices $\Gamma_{Y}$ and $\underline{\gamma}_{y x}$ in the relation (6). For instance, with the pre-windowed method, the Yule-Walker equations (6) become the equations (12) below. Now, when the optimal filter is applied according to relations (4) or (8), we use the extensions at the edges of the image as indicated in Subsection 2.7 .
} 
signals given in [10] do not apply here. So, we present in Appendix the details of the proposed method, it is an extension to the 2-D case of the pre-windowed method given in [27] and we show that the computation of the matrix $\mathcal{Y} \mathcal{Y}^{T}$ costs $2[2(4 q+1)(3 p+1)+1] M_{2} N_{2}+o\left(M_{2} N_{2}\right)$ arithmetic operations.

\subsection{Locally adapted estimation (LAE) method}

\subsubsection{Choice of the criterion}

The input signal is supposed to be a realization of a 2-D stochastic process, whose statistics of orders 1 and 2 are locally stationary. We apply then the Recursive Least Squares (RLS) algorithm. For this, we introduce a forgetting factor $\alpha, 0<\alpha \ll 1$, and the criterion to be minimized (that is the local estimation of the variance of the prediction error) is equal to

$$
J_{2}(m, n)=\sum_{i=0}^{m-1} \sum_{j=0}^{N_{2}-1} \alpha^{N_{2}(m-i)+n-j} x_{h}(i, j)^{2}+\sum_{j=0}^{n} \alpha^{n-j} x_{h}(m, j)^{2}
$$

with

$$
x_{h}(i, j)=x_{2}(i, j)-\underline{c}^{T}(m, n) \underline{y}(i, j) .
$$

We can notice in this relation that we associated the index $(m, n)$ with the vector $\underline{c}$ and the index $(i, j)$ with the other variables. It is due to the nature of the optimization: for each $(m, n), \underline{c}(m, n)$ depends on $\underline{y}(i, j)$ and $x_{2}(i, j)$ for $0 \leq i<m$ and $0 \leq j<N_{2}$ and also for $i=m$ and $0 \leq j \leq n$. The forgetting factor is applied in a way specific to the method of scanning the image (from left to right and from top to bottom).

For the LAE method, the supports $\Delta_{1}$ and $\Delta_{2}$ of the filters used in the relations (4) and (8) are subsets of $\Delta_{1}^{\prime}$ and $\Delta_{2}^{\prime}$ given respectively in the relations (9) and (10). Indeed, in an image the pixels which contain the most useful information for the estimation of the current pixel are generally its closest neighbors. It is thus natural to reorder the components of vectors $\underline{y}_{1}(m, n)$ and $\underline{y}_{2}(m, n)$, so that their new order is an increasing function of the distance to the current pixel. Therefore, only $r_{1}$ coefficients of $\Delta_{1}^{\prime}$ are retained, those numbered from 1 to $r_{1}$ in the left diagram of Fig. 5 , and only $r_{2}$ coefficients of $\Delta_{2}^{\prime}$ are retained, those numbered from 1 to $r_{2}$ on the right diagram of Fig. 5. So, it is now possible to decrease the order of the filters and consequently the computing time without reducing the performances.

Using the relation (14) in the equation (13), the criterion $J_{2}$ can be expressed as:

$$
J_{2}(m, n)=\kappa(m, n)-2 \underline{c}(m, n)^{T} \underline{\Theta}(m, n)+\underline{c}(m, n)^{T} \mathbf{\Phi}(m, n) \underline{c}(m, n)
$$


with $\kappa(m, n)=\sum_{i=0}^{m-1} \sum_{j=0}^{N_{2}-1} \alpha^{N_{2}(m-i)+n-j} x_{2}(i, j)^{2}+\sum_{j=0}^{n} \alpha^{n-j} x_{2}(m, j)^{2}$ and

$$
\begin{aligned}
& \underline{\Theta}(m, n)=\sum_{i=0}^{m-1} \sum_{j=0}^{N_{2}-1} \alpha^{N_{2}(m-i)+n-j} x_{2}(i, j) \underline{y}(i, j)+\sum_{j=0}^{n} \alpha^{n-j} x_{2}(m, j) \underline{y}(m, j) \\
& \boldsymbol{\Phi}(m, n)=\sum_{i=0}^{m-1} \sum_{j=0}^{N_{2}-1} \alpha^{N_{2}(m-i)+n-j} \underline{y}(i, j) \underline{y}(i, j)^{T}+\sum_{j=0}^{n} \alpha^{n-j} \underline{y}(m, j) \underline{y}(m, j)^{T} .
\end{aligned}
$$

The criterion $J_{2}(m, n)$ can now be minimized by cancelling its partial derivatives with respect to $\underline{c}(m, n)$ : $\frac{\partial J_{2}(m, n)}{\partial \underline{c}(m, n)}=-2 \underline{\Theta}(m, n)+2 \boldsymbol{\Phi}(m, n) \underline{c}(m, n)$. We obtain the Yule-Walker equations:

$$
\boldsymbol{\Phi}(m, n) \underline{c}(m, n)=\underline{\Theta}(m, n)
$$

which have the solution $\underline{\hat{\hat{c}}}(m, n)=\boldsymbol{\Phi}(m, n)^{-1} \underline{\underline{\Theta}}(m, n)$. From the relations (16) and (17) we deduce the recursive expressions ${ }^{3}$

$$
\begin{aligned}
& \boldsymbol{\Phi}(m, n)=\alpha \boldsymbol{\Phi}(m, n-1)+\underline{y}(m, n) \underline{y}(m, n)^{T} \\
& \underline{\Theta}(m, n)=\alpha \underline{\Theta}(m, n-1)+x_{2}(m, n) \underline{y}(m, n)^{T} .
\end{aligned}
$$

The RLS algorithm expresses $\boldsymbol{\Phi}^{-1}(m, n)$ in terms of $\boldsymbol{\Phi}^{-1}(m, n-1)$ in such a way that the equation (18) can be solved without the inversion of the matrix $\mathbf{\Phi}(m, n)$ at each step $(m, n)$. For this, the matrix inversion lemma [28]: $\left(\mathbf{S}^{-1}+\mathbf{U V}^{-1} \mathbf{U}^{T}\right)^{-1}=\mathbf{S}-\mathbf{S U}\left[\mathbf{V}+\mathbf{U}^{T} \mathbf{S} \mathbf{U}\right]^{-1} \mathbf{U}^{T} \mathbf{S}$ is applied, with $\mathbf{S}^{-1}=\alpha \mathbf{\Phi}(m, n-1)$, $\mathbf{U}=\underline{y}(m, n)$ and $\mathbf{V}^{-1}=1$. That leads to

$$
\boldsymbol{\Phi}^{-1}(m, n)=\alpha^{-1} \boldsymbol{\Phi}^{-1}(m, n-1)-\frac{\alpha^{-1} \boldsymbol{\Phi}^{-1}(m, n-1) \underline{y}(m, n) \underline{y}(m, n)^{T} \boldsymbol{\Phi}^{-1}(m, n-1)}{\alpha+\underline{y}(m, n)^{T} \boldsymbol{\Phi}^{-1}(m, n-1) \underline{y}(m, n)}
$$

This relation requires an initial value $\mathbf{\Phi}(1,1)$. The simplest way is to take it equal to $\delta \mathbf{I}$, where $\delta$ is a small constant. Let $\underline{g}(m, n)$ be the vector

$$
\underline{g}(m, n)=\frac{\boldsymbol{\Phi}^{-1}(m, n-1) \underline{y}(m, n)}{\alpha+\underline{y}(m, n)^{T} \mathbf{\Phi}^{-1}(m, n-1) \underline{y}(m, n)}
$$

the equation (21) becomes $\boldsymbol{\Phi}^{-1}(m, n)=\alpha^{-1} \boldsymbol{\Phi}^{-1}(m, n-1)-\alpha^{-1} \underline{g}(m, n) \underline{y}(m, n)^{T} \boldsymbol{\Phi}^{-1}(m, n-1)$ and the equation (22) can be changed into $\underline{g}(m, n)=\boldsymbol{\Phi}^{-1}(m, n) \underline{y}(m, n)$. This vector is called the adaptation gain, since it appears as a gain applied to the error of prediction in the equation that updates $\underline{\hat{c}}(m, n)$ : $\underline{\hat{c}}(m, n)=\underline{\hat{c}}(m, n-1)+\underline{g}(m, n)\left[x_{2}(m, n)-\underline{\hat{c}}^{T}(m, n-1) \underline{y}(m, n)\right]$. The procedure of adaptive decomposition is summarized in Tab. 1.

\footnotetext{
${ }^{3}$ Similar expressions can be obtained by replacing the indexes $(m, n)$ with $(m, 0)$ and $(m, n-1)$ with $\left(m-1, N_{2}-1\right)$; this corresponds to the change of row of the current pixel.
} 


\subsubsection{Complexity of the method}

The complexity ${ }^{4}$ of the LAE method is $O\left(M_{2} N_{2}\left(r_{1}+r_{2}\right)^{2}\right)[28]$ for one level of decomposition. Let us notice that this procedure of decomposition uses the 1-D RLS algorithm [28]. Nevertheless, the construction of the observation vector $\underline{y}(m, n)$, where the bi-dimensional neighborhood of sample $x_{2}(m, n)$ appears, gives a 2-D characteristic to this algorithm, that is why we kept the indexes $(m, n)$ in the notation of the vectors.

\subsection{Comparison of the two LSE methods}

The major difference between the two methods is that the filter coefficients must be transmitted to the decoder with the GAE, but not with the LAE. Indeed, with the GAE method, the optimal filter is computed only during the coding, whereas with the LAE method, it is estimated for each pixel during both the coding and the decoding. This is why we obtain such a difference between the mean decoding times of the two methods (see Tab. 6). Another difference is that the computation of the optimal filter requires an extension at the edges of the image only with the GAE. The major similarity between GAE and LAE is that they minimize each the variance of the residue of estimation. It is the estimation of this variance that differs according to the stationarity assumptions made on the image: global or local. However, it is well known that the minimization of the variance is optimal for coding Gaussian data and is not optimal generally when the data are not Gaussian. Another important similarity is that included in a multi-resolution framework, each method permits progressive coding in resolution. Let us notice that since the decoder knows the filters coefficients in the GAE method (only), man could use the integer-to-integer generalized lifting scheme with GAE for a progressive coding in quality (or rate). Now it is not the case, because the criterion used does not take into account the distortion and is justified only for lossless coding. We emphasize that the problem for a progressive coding in rate is due to the choice of the adaptation criterion and not to the structure of the generalized lifting scheme.

\footnotetext{
${ }^{4}$ We did not implement a fast version (see [30]) of the RLS algorithm.
} 


\section{Application to lossless coding of synthetic images}

In this section we estimate the performances of one predict step (at the highest resolution) of the integer-tointeger generalized lifting scheme on artificial images that satisfy the assumptions of an optimal coding and we compare with other integer-to-integer decompositions.

\subsection{Construction of the synthetic images}

In our experiments, we used two families of Gaussian synthetic images, coded on 8 bits per pixel (bpp). The first one is composed of eight 2-D Auto-Regressive (AR) signals of size $512 \times 512$, which are each globally WSS. Half of these signals are generated with 2-D AR models which have a quarter-plan (QP) causality and the other half with a NSHP causality. They are shown in Fig. 6. The second family is a set of eight images of size $512 \times 512$, composed of several areas with different textures, which are each 2-D AR signals, simulating local stationarity (i.e., each area is stationary). The textures of the different areas have been generated with 2-D AR models having either QP or NSHP causality (chosen at random). They are shown on Fig. 6.

\subsection{Experimental results}

In this subsection, we compare for each synthetic image the variance and the first order entropy of subband $x_{h}$, obtained with the reversible version of the diagram in Fig. 3 (described in $\S 2.4$ ), with the GAE method ( $p=3, q=3$, separable decimation), LAE method $\left(r_{1}=16, r_{2}=8, \alpha=0.9995\right.$, separable decimation $)$, the reversible discret wavelet decompositions (DWT) $(9,7)$ and $(5,3)$ of Daubechies and the method by Gerek \& Çetin $[31,11]$ mentioned above. Tab. 2 and Tab. 3 present the performances of the different decompositions. For the two families of signals, the performances of the decompositions GAE and LAE are quite higher than those of the other decompositions and very close to one another. Indeed, on average, the gain is of $0.5 \mathrm{bpp}$ for the globally WSS signals or $0.7 \mathrm{bpp}$ for the locally stationary signals compared to the best of the other decompositions. We notice, as it was expected, that for globally stationary signals the GAE method gives slightly better results than LAE and that for locally stationary signals it is the LAE method which slightly precedes GAE. We also notice that the addition of the filter $B\left(z_{1}, z_{2}\right)$ appreciably improves (0.6 or 0.7 bpp on average) the performances of the adapted lifting scheme, thanks to a comparison with the 
method of Gerek \& Çetin.

\section{Comparative evaluations in lossless image compression}

In this section, we compare the performances in lossless coding of the GAE and LAE methods with other wellknown codecs: S+P with arithmetic coding [5], LOCO I [19] (that is the JPEG-LS standard), CALIC [21] and JASPER [23] (that is the JPEG2000 standard) on actual images.

\subsection{Description of the images used in our tests}

In our experiments, we considered four families of images: 1) fourteen natural images of resolutions $512 \times 512$ and $1024 \times 1024,2)$ fourteen satellite ${ }^{5}$ images of various resolutions, 3) sixteen MRI medical images of resolution $512 \times 512$ and 4) eleven images of textures and fingerprints whose resolution is either $512 \times 512$ or $768 \times 768$. All the images are coded with 8 bpp. In our tests, all the multi-resolution decompositions applied to images of resolution $512 \times 512$ or $768 \times 768$ (resp. $1024 \times 1024$ ) use five (resp. six) levels of decomposition. Some images are shown on Fig. 7

\subsection{Description of the codecs associated with the LAE and GAE methods}

For GAE and LAE methods, at each level of decomposition, meta parameters must be fixed, such as the type of 2-fold decimation (quincunx or separable), the orders of filters $A\left(z_{1}, z_{2}\right)$ and $B\left(z_{1}, z_{2}\right)$, the use of the $S$-transform or not (see subsection 2.6) and, for LAE only, the value of the forgetting factor. For each family of images and for each level of decomposition, we looked for the best values of these meta parameters, i.e., the values that give in average the lowest first order entropy. We noted that these meta-parameters vary from one family of images to another, whereas they slightly vary in the same family. Moreover, for the GAE method we noted that when the resolution is not greater than $64 \times 64$, in the adapted predict step, it is better to apply a process close to the LAR method [32] than to use the linear estimator described above, since there are not enough samples for a good estimation of the second order statistics. The process then consists in dividing the image of the subband $x_{1}(m, n)$ in homogenous areas and in evaluating the estimation

\footnotetext{
${ }^{5}$ The satellite images have been given as a favor by the French National Center of Spatial Studies (CNES).
} 
$\hat{x}_{2}(m, n)$ as the average of the pixels $x_{1}(m, n)$ which belong to the same homogenous area. The variant of GAE obtained by adapting the meta-parameters to each family of images is noted GAEa in Tab. 5 .

After the multi-resolution decomposition based on either the GAE or the LAE method, we applied a contextual adaptive arithmetic coder with one context per subband (we used the C sources $S+P$ image compression by Said [5]). To rebuild the image, the decoder needs a heading in the bit stream containing the image size, the image mean, the number of levels of decomposition and, for each level of decomposition, the orders of the filters, the filters coefficients (for GAE only), a boolean specifying the type of downsampling and another boolean specifying whether the $S$-transform has been used or not. To reduce the size of the bit stream required for coding the heading, we truncated the filter coefficients (before applying the decomposition): a coefficient $a$ is replaced by $\hat{a}=\left(\left\lfloor a \cdot 10^{s}+0.5\right\rfloor\right) \cdot 10^{-s}$ (in our experiments, we chose $s=6$ ) and the integer $10^{s} . \hat{a}$ is stored in the heading (for GAE only).

\subsection{Experimental results and analysis}

\subsubsection{Mean first order entropy}

We first compare the performances of the LAE and GAE decompositions with other integer-to-integer wavelet decompositions, without taking into account neither the bit stream required for coding the heading nor the entropic coder applied on the transformed coefficients. For this, we estimate the average of the first order entropy of the subbands, weighted by the ratio of the subband size on the entire image size. In order to be brief, the results of a few images is shown in Tab. 4, however the row "Average" corresponds, for each family of images described in Subsection 5.1, to the average bit-rate of the whole family. The columns $(a, b)$, where $a$ and $b$ are integers, correspond to integer-to-integer wavelets decompositions defined in [3]. In column $\mathrm{S}+\mathrm{P}$, the best predictor among $\mathrm{A}, \mathrm{B}$, and $\mathrm{C}$ of the transform $\mathrm{S}+\mathrm{P}[5]$ has been chosen for each image.

We notice that for relatively smooth images (containing large areas of texture) like Lena, Peppers, F16, the average of first order entropies associated with GAE and LAE is only slightly lower than the one obtained with the $\mathrm{S}+\mathrm{P}$ transformation. For images containing more outlines, like Goldhill, Barbara and the fingerprints Finger1-3, the proposed methods give the smallest average of first order entropies, the gain being approximatively 0.15 bpp. 


\subsubsection{Actual bit-rate}

We also compared the performances in lossless coding of the proposed methods on the actual bit-rate obtained with the contextual adaptive arithmetic coder implemented by Said [18]. Moreover, the bit-stream required for coding the heading is taken into account in the bit-rate. The results are shown in Tab. 5. For natural images, CALIC gives on average the smallest bit-rate, however, among codecs that permit progressive coding in resolution, the LAE method is that which gives the smallest bit-rate, slightly lower than the one of $\mathrm{S}+\mathrm{P}$ or LOCO. Jasper is higher than other codecs by about $0.1 \mathrm{bpp}$. Whereas for MRI medical images, Jasper is significantly better. This is due to the fact that MRI medical images are smooth. The efficiency of Jasper decreases on images with steep outlines. On the family of textures and fingerprints, CALIC and LAE methods are similar and have significantly better performances than the other codecs.

On natural images and on MRI medical images, the performances in lossless coding of codecs based on the proposed methods do not exceed those of classical codecs based on multiresolution decomposition with filters having fixed coefficients. However, as expected, the variance of the error of prediction $x_{h}(m n$,$) is$ smaller with the proposed methods than with the others. That illustrates the fact that the criterion of minimizing the variance for finding optimal transform in coding is only justified with Gaussian sources. For the families of satellite images and textures (with fingerprints), the codecs based on the proposed methods give a slight but still noticeable (about 0.05 to $0.08 \mathrm{bpp}$ ) coding gain compared to the others. However, the coding gain on satellite or textured images is much smaller than the one observed on synthetic images and we can deduce again that the criterion of minimizing the variance is not the good one for actual images.

\subsubsection{Scalability and complexity}

For a progressive coding in resolution, the $S$-transform is systematically applied in any 'predict' step for resolutions smaller or equal to $256 \times 256$ (in order to avoid aliasing artefacts) and we observed that, compared to the others, the positive coding gain of codecs GAEa and LAE remains noticeable at smaller resolutions, for satellite and textured images. Now, as it was mentioned in Subsection 3.3, both the GAE and LAE methods are not suitable for progressive coding in rate (or quality). In Tab. 6 we compare the coding and decoding times of the different codecs. 


\section{Conclusion}

In this paper we have introduced an adapted generalized lifting scheme, in which the predict step is built upon two filters, leading to taking advantage of all the information available at the decoder. With this structure applied in a multiresolution decomposition framework, we have studied two kinds of adaptation based on LSE, according to the different stationarity assumptions made on the input image. One decomposition, called globally adapted estimation (GAE), assumes the entire input image is a WSS signal. The other one, called locally adapted estimation (LAE), assumes only local WSS. The efficiency in lossless coding of these decompositions has been shown on Gaussian synthetic images satisfying these stationarity conditions and their performances have been compared with those of well-known codecs (S+P [18], LOCO I [20], CALIC [22] and Jasper [23]) on actual images. We have considered four families of images : natural, MRI medical, satellite and textures associated with fingerprints. On natural and MRI medical images, the performances in lossless coding of codecs based on the proposed methods do not exceed those of classical codecs. Nevertheless, for the families of satellite images and textures (with fingerprints), the codecs based on the proposed methods give a slight but still noticeable (about 0.05 to $0.08 \mathrm{bpp}$ ) coding gain compared to the others, at the price of a more important coding time. However, the coding gain for satellite and textured images is much smaller than the one observed on synthetic images and some improvements have to be done in order to satisfy the applications of satellite images. In future works we shall test other criteria, associated with the generalized lifting scheme, based on mutual information as the ones clarified in [33] or in [34].

\section{Acknowledgment}

The authors are grateful to Pierre Duhamel and anonymous reviewers for their very helpful comments as well as Jean-Luc Collette and Jean-Louis Gutzwiller who took part in the simulation work.

\section{A Fast calculation of the Yule-Walker equations}

First we introduce new notations: for two vectors $\underline{u} \in \mathbb{R}^{\alpha}$ and $\underline{v} \in \mathbb{R}^{\beta}$, we denote $\mathbf{T}[\underline{u}, \underline{v}]$ the Toeplitz matrix of dimension $\alpha \times \beta$ whose first column (resp. row) is equal to $\underline{u}$ (resp. $\underline{v}^{T}$ ). For a matrix $\mathbf{A}$ of dimension 
$m \times n$, the element localized at the intersection of the $(i+1)^{\text {th }}$ row and the $(j+1)^{\text {th }}$ column $(0 \leq i<m$ and $0 \leq j<n)$ is denoted by $[\mathbf{A}]_{i, j}$. Let us introduce the vector $\underline{b}_{0}=\left(0, \ldots, 0,-1, b_{0,1}, b_{0,2}, \ldots, b_{0, q}\right)^{T}$ of dimension $2 q+1$, the $p$ vectors $\underline{b}_{\ell}=\left(b_{\ell,-q}, b_{\ell,-q+1}, \ldots, b_{\ell, q}\right)^{T}(1 \leq \ell \leq p)$ of dimension $2 q+1$, the $2 p+1$ vectors $\underline{a}_{\ell}=\left(a_{\ell,-q}, a_{\ell,-q+1}, \ldots, a_{\ell, q}\right)^{T}(-p \leq \ell \leq p)$ of dimension $2 q+1$ and the vectors $\underline{b}^{\prime}=\left(\underline{b}_{0}^{T}, \underline{b}_{1}^{T}, \ldots, \underline{b}_{p}^{T}\right)^{T}$ and $\underline{a}=\left(\underline{a}_{-p}^{T}, \underline{a}_{-p+1}^{T}, \ldots, \underline{a}_{p}^{T}\right)^{T}$. With the $(m+1)^{\text {th }}$ row of the subband signal $x_{2}$, we associate the $p+1$ Toeplitz matrices $(0 \leq \ell \leq p)$

$$
\mathbf{X}_{\ell}(m)=\mathbf{T}\left[\left(x_{2}(m-\ell, q), \ldots, x_{2}\left(m-\ell, N_{2}-1\right)\right)^{T},\left(x_{2}(m-\ell, q), \ldots, x_{2}(m-\ell, 0), 0, \ldots, 0\right)^{T}\right]
$$

of dimension $\left(N_{2}-q\right) \times(2 q+1)$ and the block matrix

$$
\mathbf{X}(m)=\left[\begin{array}{llll}
\mathbf{X}_{0}(m) & \mathbf{X}_{1}(m) & \cdots & \mathbf{X}_{p}(m)
\end{array}\right]
$$

of dimension $\left(N_{2}-q\right) \times(2 q+1)(p+1)$. In the same way, with the $(m+1)^{\text {th }}$ row of the signal $x_{1}$ we associate the $2 p+1$ Toeplitz matrices $(-p \leq \ell \leq p)$

$$
\mathbf{Y}_{\ell}(m)=\mathbf{T}\left[\left(x_{1}(m-\ell, q), \ldots, x_{1}\left(m-\ell, N_{2}-1\right)\right)^{T},\left(x_{1}(m-\ell, q), \ldots, x_{1}(m-\ell, 0), 0, \ldots, 0\right)^{T}\right]
$$

of dimension $\left(N_{2}-q\right) \times(2 q+1)$ and the block matrix $\mathbf{Y}(m)=\left[\mathbf{Y}_{-p}(m) \mathbf{Y}_{-p+1}(m) \cdots \mathbf{Y}_{p}(m)\right]$ of dimension $\left(N_{2}-q\right) \times(2 p+1)(2 q+1)$. With these notations, the error of estimation $\underline{x}_{h}(m)=\left[x_{h}(m, 0), \ldots, x_{h}\left(m, N_{2}-\right.\right.$ $q-1)]^{T}$ associated to the $(m+1)^{\text {th }}$ row of $x_{2}$ satisfies the relation $-\underline{x}_{h}(m)=\mathbf{X}(m) \underline{b}^{\prime}+\mathbf{Y}(m) \underline{a}$. Moreover, it results from the relations $(23)$ and $(25)$ that $\mathbf{Y}_{k}(m)=\mathbf{Y}_{k-1}(m-1)=\mathbf{Y}_{0}(m-k), \mathbf{X}_{k}(m)=\mathbf{Y}_{k}(m)=\mathbf{0}$ (if $m<k$ ) and that for $0<k \leq p$ and $0 \leq m<M_{2}-p$

$$
\mathbf{X}_{k}(m)=\mathbf{X}_{k-1}(m-1)=\mathbf{X}_{0}(m-k)
$$

The equations of estimation lead then to

$$
-\left(\underline{x}_{h}(0)^{T}, \underline{x}_{h}(1)^{T}, \ldots, \underline{x}_{h}\left(M_{2}-p-1\right)^{T}\right)^{T}=\mathbf{X} \underline{b}^{\prime}+\mathbf{Y} \underline{a},
$$

with the block Toeplitz matrices $\mathbf{X}$ and $\mathbf{Y}$, whose first columns are respectively $\left(\mathbf{X}_{0}(0)^{T}, \mathbf{X}_{0}(1)^{T}, \ldots, \mathbf{X}_{0}\left(M_{2}-\right.\right.$ $\left.p-1)^{T}\right)^{T}$ and $\left(\mathbf{Y}_{0}(p)^{T}, \mathbf{Y}_{0}(p+1)^{T}, \ldots, \mathbf{Y}_{0}\left(M_{2}-1\right)^{T}\right)^{T}$ and whose first rows are respectively $\left(\mathbf{X}_{0}(0), \mathbf{0}, \ldots, \mathbf{0}\right)$ and $\left(\mathbf{Y}_{0}(p), \mathbf{Y}_{0}(p-1), \ldots, \mathbf{Y}_{0}(0), \mathbf{0}, \ldots, \mathbf{0}\right)$. Since each block $\mathbf{X}_{0}(k)$ or $\mathbf{Y}_{0}(k)$ is Toeplitz, we may observe that both the matrices $\mathbf{X}$ are $\mathbf{Y}$ are Toeplitz and also block Toeplitz. The coefficients of the optimal filters 
are solutions to the Yule-Walker equations [28] that may be written $\left(\underline{b}^{T}, \underline{a}^{T}\right) \mathbf{C}=\left(\underline{u}^{T},-W, \underline{0}^{T}\right)$, where $\underline{u}$ is a vector of dimension $q, W=\left(M_{2}-p\right)\left(N_{2}-q\right) J_{1}$ and

$$
\mathbf{C}=\left[\begin{array}{ll}
\mathbf{X} & \mathbf{Y}
\end{array}\right]^{T}\left[\begin{array}{ll}
\mathbf{X} & \mathbf{Y}
\end{array}\right]=\left[\begin{array}{cc}
\mathbf{X}^{T} \mathbf{X} & \mathbf{X}^{T} \mathbf{Y} \\
\mathbf{Y}^{T} \mathbf{X} & \mathbf{Y}^{T} \mathbf{Y}
\end{array}\right]
$$

is a symmetrical matrix of order $\omega^{\prime}=(2 q+1)(3 p+2)$. Since the $q+1$ first components of $\underline{b}^{\prime}$ are known, the actual dimension of the system is $\omega=6 p q+3 p+3 q+1$. However, it is faster to calculate first the entire matrix $\mathbf{C}$ and then to extract from it the system's matrix $\mathcal{Y}^{T} \mathcal{Y}$, than to directly calculate this last matrix. The relation (27) differs from the well known equations encountered with the pre-windowed method [27] [28] and from the equations in [10] in the extra terms $\mathbf{Y} \underline{a}$. Consequently, the displacement rank [27] of the matrix $\mathbf{C}$ is not equal to 1 . Nevertheless, the elements of $\mathbf{C}$ still have a great redundancy and the reasoning presented in [29] can be adapted to this matrix.

To be short, only the relations that permit to calculate the block $\mathbf{X}^{T} \mathbf{X}$ of $\mathbf{C}$ are given. The same reasoning could be carried out for the other blocks. It results from the relations (27) and (24) that $\mathbf{X}^{T} \mathbf{X}=$ $\sum_{m=0}^{M_{2}-p-1} \mathbf{X}(m)^{T} \mathbf{X}(m)=\left[\sum_{m=0}^{M_{2}-p-1} \mathbf{X}_{k}(m)^{T} \mathbf{X}_{\ell}(m)\right]$ (for $0 \leq k, \ell<p$ ), where the last expression is a block representation of $\mathbf{X}^{T} \mathbf{X}$. Let $\mathbf{T}_{k, \ell}=\sum_{m=0}^{M_{2}-p-1} \mathbf{X}_{k}(m)^{T} \mathbf{X}_{\ell}(m)(0 \leq k, \ell<p)$ be the block of dimension $(2 q+1) \times(2 q+1)$. We have $\mathbf{T}_{k, \ell}^{T}=\mathbf{T}_{\ell, k}$. The relation (26) leads to $\mathbf{T}_{k, \ell}=\mathbf{T}_{k-1, \ell-1}-\mathbf{X}_{0}\left(M_{2}-p-\right.$ $k)^{T} \mathbf{X}_{0}\left(M_{2}-p-\ell\right)$ for $1 \leq k \leq \ell \leq p$. It is straightforward to deduce from (23) that $\mathbf{X}_{0}(m-k)^{T} \mathbf{X}_{0}(m-\ell)$ $\left(0 \leq k \leq \ell \leq p\right.$ and $\left.\ell \leq m<M_{2}-p\right)$ has a displacement rank of 2 . Therefore, for $0 \leq \ell \leq p$, we have $\left[\mathbf{T}_{0, \ell}\right]_{i, j}=\left[\mathbf{T}_{0, \ell}\right]_{i-1, j-1}+\sum_{m=\ell}^{M_{2}-p-1} x_{2}(m, q-i) x_{2}(m-\ell, q-j)-\sum_{m=\ell}^{M_{2}-p-1} x_{2}\left(m, N_{2}-i\right) x_{2}\left(m-\ell, N_{2}-j\right)$. In conclusion, the calculation of a block requires (in additions and multiplications):

- $O\left(N_{2}\right)$ operations for $\mathbf{T}_{k, \ell}$ with $0 \leq k \leq \ell \leq p$,

- $2(4 q+1) M_{2} N_{2}+o\left(M_{2} N_{2}\right)$ operations for $\mathbf{T}_{0, \ell}$ with $p \geq \ell \geq 1$,

- $2(2 q+1) M_{2} N_{2}+o\left(M_{2} N_{2}\right)$ operations for $\mathbf{T}_{0,0}$,

and the calculus of $\mathbf{X}^{T} \mathbf{X}$ requires $2[(4 q+1) p+2 q+1] M_{2} N_{2}+o\left(M_{2} N_{2}\right)$ operations. In the same way, the computation of $\mathbf{X}^{T} \mathbf{Y}$ requires $2(4 q+1)(3 p+1)+o\left(M_{2} N_{2}\right)$ operations and the computation of $\mathbf{Y}^{T} \mathbf{Y}$ requires $2[(4 q+1) 2 p+2 q+1] M_{2} N_{2}+o\left(M_{2} N_{2}\right)$ operations. Finally, the computation of $\mathbf{C}$ costs $2[2(4 q+1)(3 p+1)+$ 1] $M_{2} N_{2}+o\left(M_{2} N_{2}\right)$ operations. 


\section{References}

[1] M. Antonini, M. Barlaud, P. Mathieu and I. Daubechies, "Image coding using wavelet transform", IEEE Trans. on Image Processing, vol. 1, no. 2, pp. 205-219, Apr. 1992.

[2] W. Sweldens, "The lifting Scheme: A new phylosophy in biorthogonal wavelet construction", in Proc. SPIE, vol 2569, pp. 68-78, Sept. 1995.

[3] A. R. Calderbank, I. Daubechies, W. Sweldens and B. L. Yeo, "Wavelet transforms that map integers to integers", Appl. Comput. Harmon. Anal., vol. 3, no. 2, pp. 186-200, 1996. IEEE Trans. Image Processing, vol. 10, no. 1, pp. 1-14, Jan. 2001.

[4] I. Daubechies and W. Sweldens, "Factoring wavelet transforms into lifting steps", J. Fourier Anal. Appl., vol. 4, no. 3, pp. 247-269, 1998.

[5] A. Said and W. A. Pearlman, "An image multiresolution representation for lossless and lossy compression", IEEE Trans. Image Processing, vol. 5, pp. 1303-1310, Sep. 1996.

[6] M. D. Adams and F. Kossentini, "Reversible Integer-to-Integer Wavelet Transforms for Image Compression: Performance Evaluation and Analysis", IEEE Trans. Image Processing, vol. 9, no. 6, pp. 1010-1024, Jun. 2000.

[7] F. J. Hampson and J. C. Pesquet, " $M$-band nonlinear subband decomposition with perfect decomposition", IEEE Trans. Image Processing, vol. 7, no. 11, pp. 1547-1560, Nov. 1998.

[8] I. Amonou and P. Duhamel, "Nonredundant representation of images allowing object- based and multiresolution scalable coding", in Proc. Int. Conf. Visual Communications and Image Processing, pp. 598-608, Perth, Australia, Jun. 2000.

[9] Y.-S. Chung and M. Kanefsky, "On 2D recursive LMS algorithms using ARMA prediction for ADPCM encoding of Images", IEEE Trans. Image Processing, vol. 1, no. 3, pp. 416-422, Jul. 1992.

[10] X. Wu, K. U. Barthel and W. Zhang, "Piecewise 2D Autoregression for Predictive Image Coding", Proc. of the IEEE Int. Conf. on Image Processing, vol. 3, pp. 901-904, Chicago (USA), Oct. 1998. 
[11] Ö. N. Gerek and A. E. Çetin, "Adaptive polyphase subband decomposition structures for image compression", IEEE Trans. on Image Proc., 9(10):1649-1660, oct. 2000.

[12] N. V. Boulgouris, D. Tzovaras and M. G. Strintzis, "Lossless image compression based on optimal prediction, adaptive lifting, and conditional arithmetic coding", IEEE Trans. Image Processing, vol. 10, no. 1, pp. 1-14, Jan. 2001.

[13] R. L. Claypoole, G. M. Davis, W. Sweldens and R. Baraniuk, "Nonlinear wavelet transform for image coding via lifting", IEEE Trans. on Image Processing, vol. 2, no. 12, pp. 1449-1459, Dec. 2003.

[14] A. Gouze, A. Antonini, M. Barlaud and B. Macq, "Optimized lifting scheme for two-dimensional quincunx sampling", Proc. of the IEEE Int. Conf. on Image Processing, vol. 2, pp. 253-256, Thessaloniki, Greece, Oct. 2001.

[15] M. Barret and H. Bekkouche, "Adapted nonlinear multiresolution decomposition with applications in progressive lossless image coding", in Proc. Int. Symp. Image and Signal Processing and Analysis, pp. 609-613, Pula, Croatia, Jun. 2001.

[16] H. Bekkouche and M. Barret, "Adaptive multiresolution decomposition: application to lossless image compression", in Proc. IEEE Int. Conf. Acoustic, Speech and Signal Processing, Orlando, Florida, USA, May 2002.

[17] H. Bekkouche and M. Barret, "Comparison of lossless codecs for satellite and MRI medical images", in Proc. XI European Signal Processing Conf., Toulouse, France, Sept. 2002.

[18] http://www.cipr.rpi.edu/research/SPIHT/EW_Code/lossless.zip

[19] M. J. Weinberger, G. Seroussi and G. Sapiro, "The LOCO-I lossless image compression algorithm: Principles and standardization into JPEG-LS", IEEE Trans. Image Processing, vol 9, pp. 1309-1324, Aug. 2000.

[20] http://www.hpl.hp.com/loco/software.htm

[21] X. Wu and N. Memon, "Context-based, Adaptive, Lossless Image Coding", IEEE Trans. Communication, vol. 45, no. 4, pp. 437-444, Apr. 1997. 
[22] http://www.csd.uwo.ca/faculty/wu/

[23] http://www.ece.ubc.ca/ ${ }^{\sim}$ mdadams/jasper/

[24] W. Sweldens, "The lifting scheme: A custom-design construction of biorthogonal wavelets", Appl. Comput. Harmon. Analysis, 3(2):186-200, 1996.

[25] S. Mallat, A Wavelet Tour of Signal Processing, Academic Press, 1999.

[26] M. Barret, Digital filters design for signal and image processing, edited by M. Najim, chap. 10: Filter stability and chap. 11: The two-dimensional domain, ISTE Ltd, 2006.

[27] B. Friedlander, M. Morf, T. Kailath and L. Ljung, "New inversion formulas for matrices classified in terms of their distance from Toeplitz matrices", Linear Algebra and its Applications, vol. 27, pp. 31-60, 1979.

[28] S. Haykin, Adaptive filter theory, Engelwood Cliffs, NJ: Prentice-Hall, second edition 1991.

[29] T. Kailath, S. Y. Kung and M. Morf, "Displacement ranks of a matrix", Bull. Amer. Math. Soc., vol. 1, no. 5, pp. 769-773, Sep. 1979.

[30] X. Liu and M. Najim, "A two-dimensional fast lattice recursive least squares algorithm", IEEE Trans. Signal Processing, vol. 44, no. 10, Oct. 1996.

[31] Ö. N. Gerek and A. E. Çetin , "Polyphase adaptive filter banks for fingerprint image compression", Proc. 9ème European Signal Processing Conference, pp. 45-48, Rhodes, Grèce, sept. 1998.

[32] O. Déforges and J. Ronsin, "Locally Adaptive Resolution method for progressive still image coding", in Proc. Int. Symp. Signal Processing and its Applications, Brisbane, Australia, 22-25 Aug. 1999.

[33] M. Narozny, M. Barret and D. T. Pham, "ICA based algorithms for computing optimal 1-D linear block transforms in variable high-rate source coding", to be published in Signal Processing.

[34] A. Benazza-Benyahia, J. C. Pesquet, J. Hattay and H. Masmoudi, "Block-based adaptive vector lifting chemes for multichannel image coding", EURASIP Journal on Image and Video Processing, vol. 2007, article ID 13421, 10 pages, 2007. 

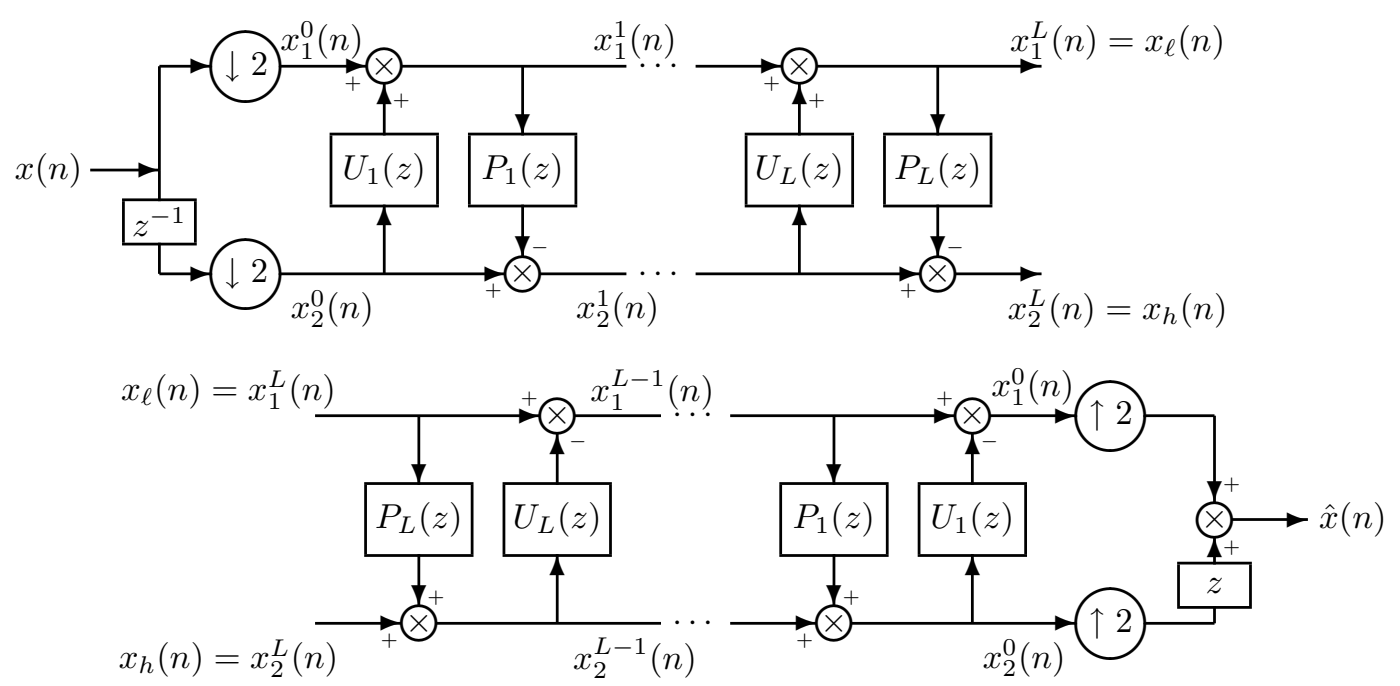

Figure 1: Standard lifting scheme for perfect reconstruction filter bank with two subbands. Top: analysis filter bank ; bottom: associated synthesis filter bank.
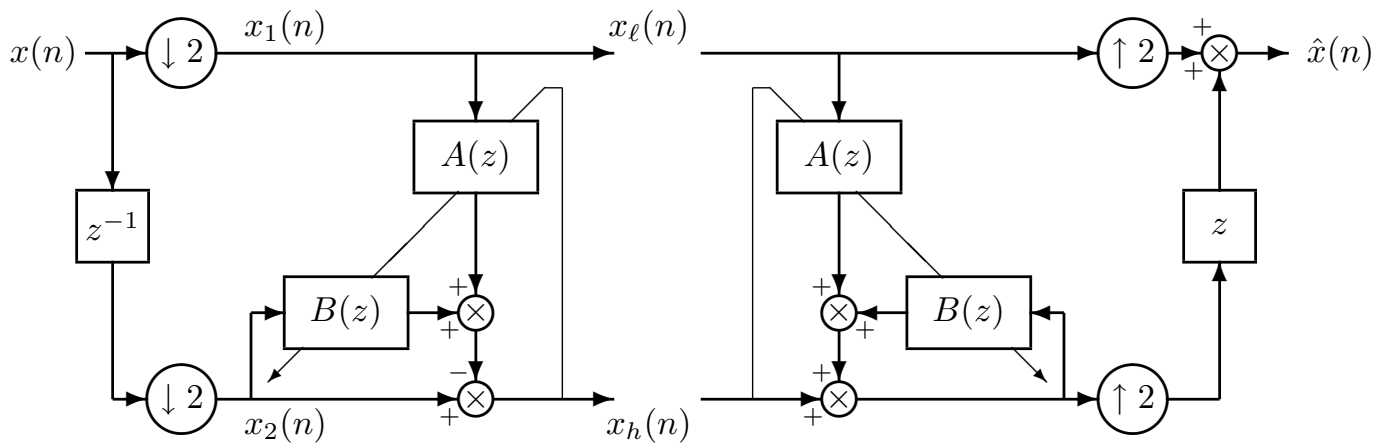

Figure 2: Predict step of the generalized lifting scheme. Compared to the standard lifting scheme, a second filter $B(z)$ is introduced. The filters $A$ and $B$ adapt to the input data.

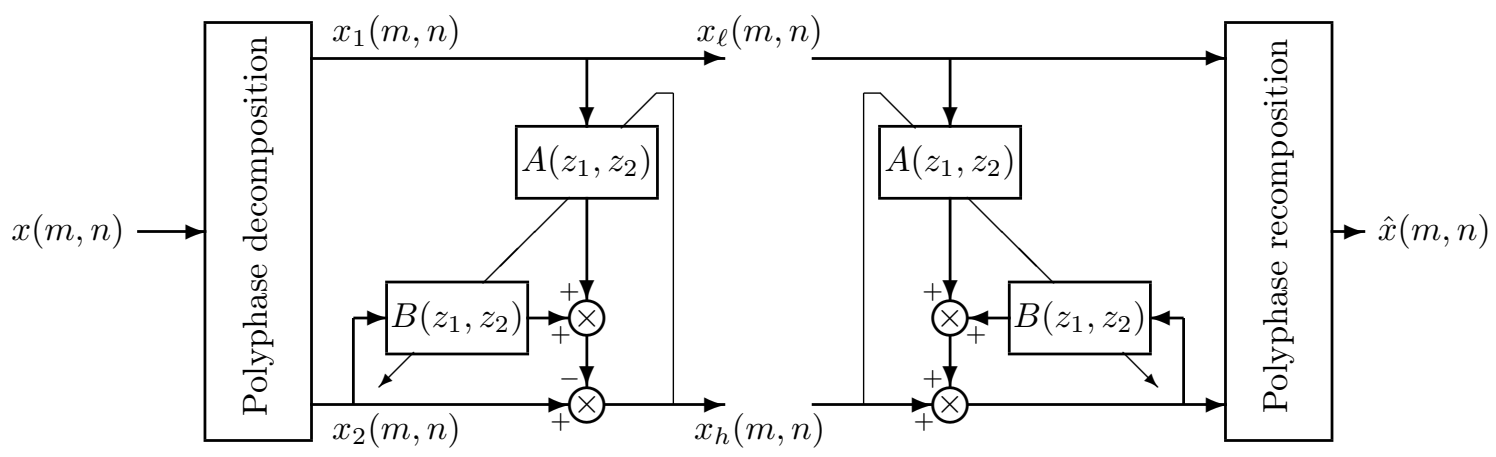

Figure 3: Predict step of the generalized lifting scheme in the 2-D case. The additional filter $B\left(z_{1}, z_{2}\right)$ is introduced in comparison with the standard lifting scheme. Each of the filters $A$ and $B$ are adapted to the input signal. 


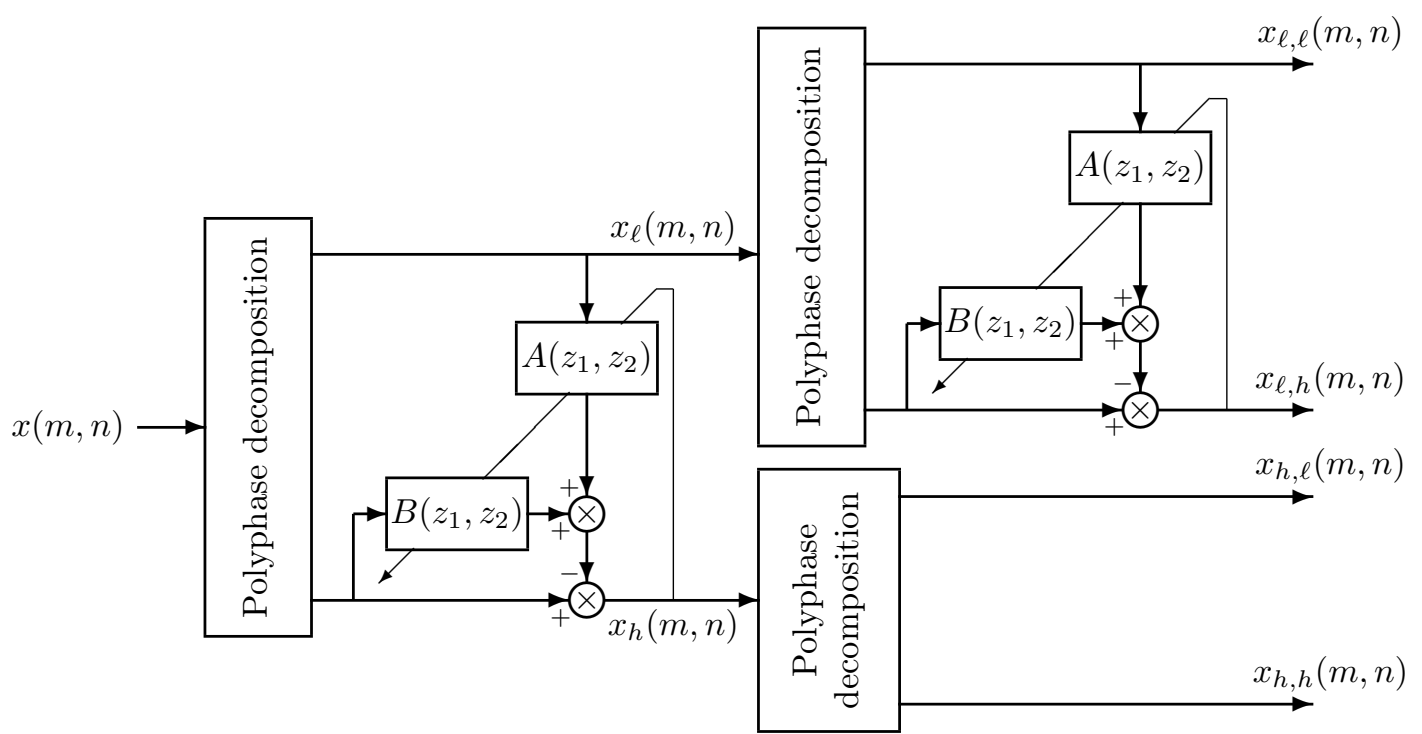

Figure 4: One level of decomposition of the image with the adapted generalized lifting scheme.

\begin{tabular}{|c|c|c|c|c|c|c|}
\hline \multicolumn{7}{|c|}{1} \\
\hline 49 & 44 & 36 & 28 & 35 & 43 & 48 \\
\hline 45 & 24 & 18 & $1 \mid 1$ & 17 & 23 & 42 \\
\hline 37 & 19 & 7 & 2 & 6 & 16 & 34 \\
\hline 29 & 12 & 3 & 1 & -5 & 10 & 27 \\
\hline 30 & 20 & 8 & 4 & 9 & 15 & 33 \\
\hline 38 & 25 & 21 & 13 & 14 & 22 & 41 \\
\hline 46 & 39 & 31 & 26 & 32 & 40 & 47 \\
\hline
\end{tabular}

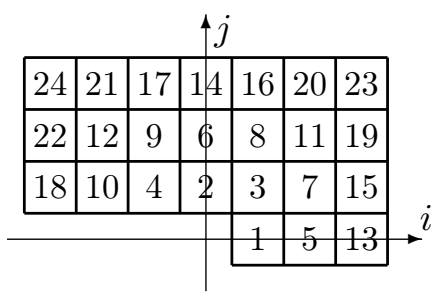

Figure 5: Order of the samples in the observation vector for $p \leq 3$; left: in $\Delta_{1}^{\prime}$; right: in $\Delta_{2}^{\prime}$ (LAE method).

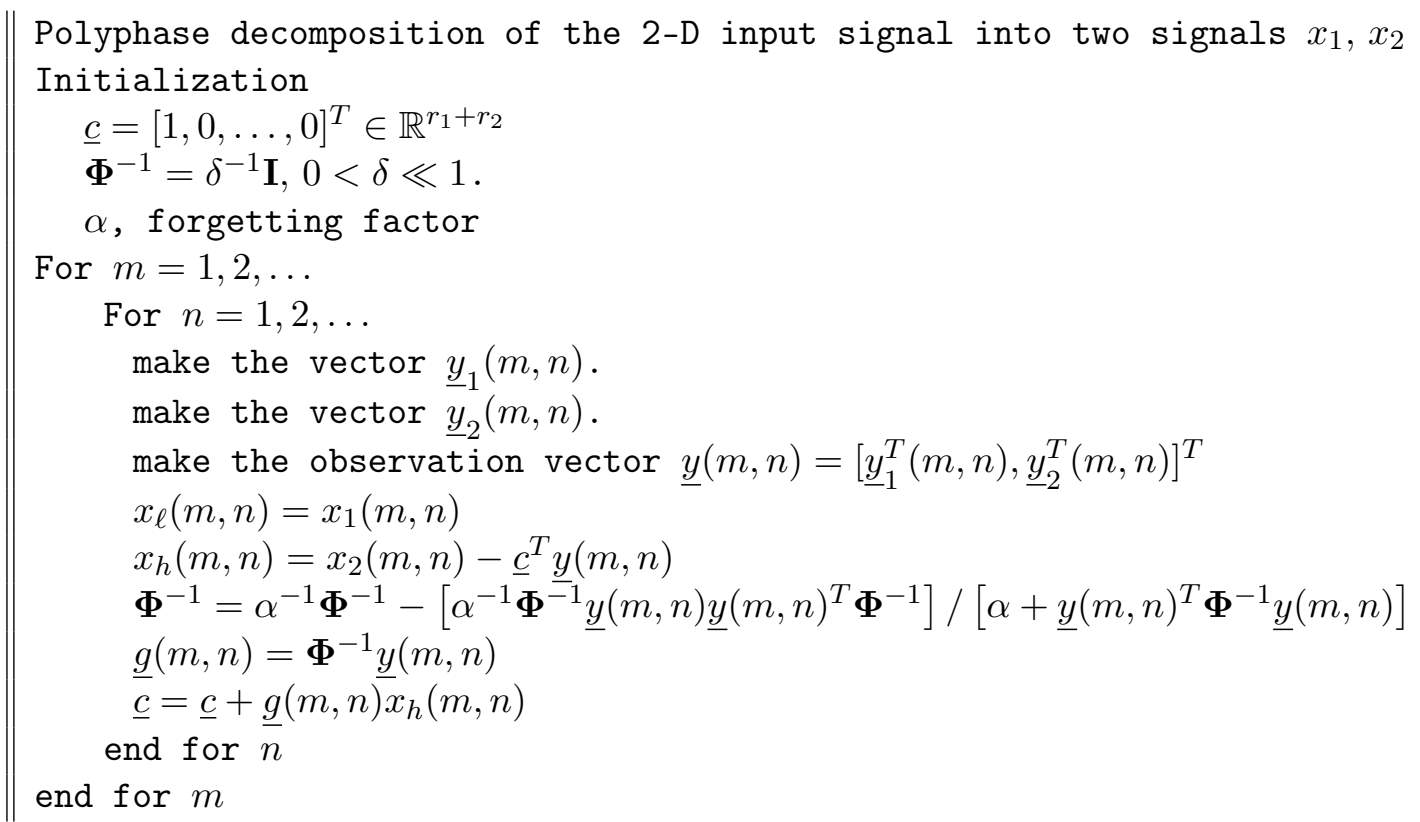

Table 1: Algorithm of adaptation of the filter coefficients for the LAE method. 


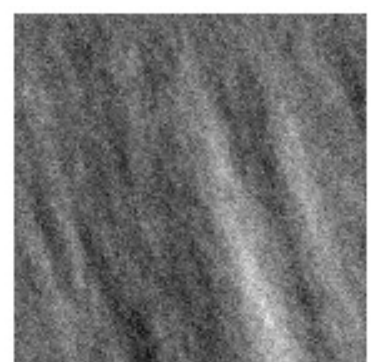

WSS 1

$(810.59 ; 6.84)$

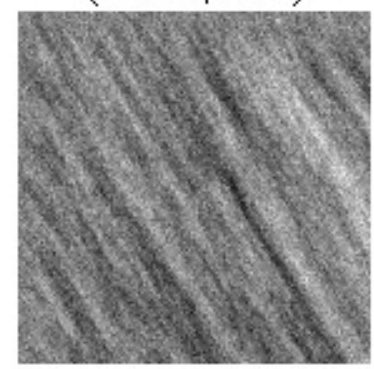

WSS_5

$(841.78 ; 6.90)$

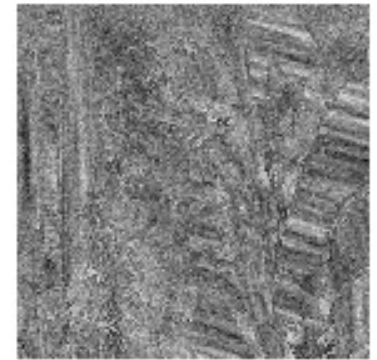

Loc WSS 1

$(86 \overline{7.85} ; 6 . \overline{93})$

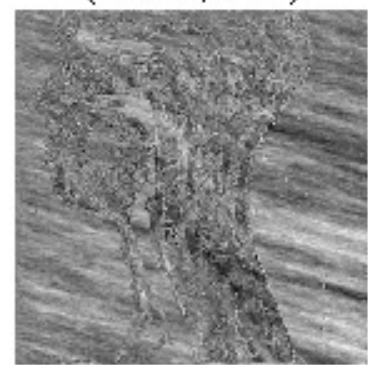

Loc_WSS_5

$(926.25 ; 6 . \overline{9})$

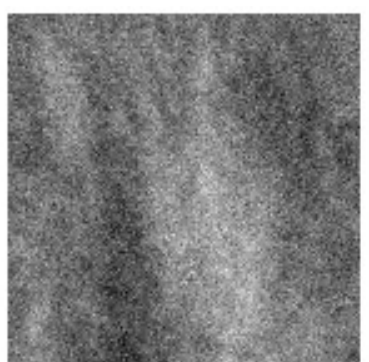

WSS 2

(886.15; 6.94)

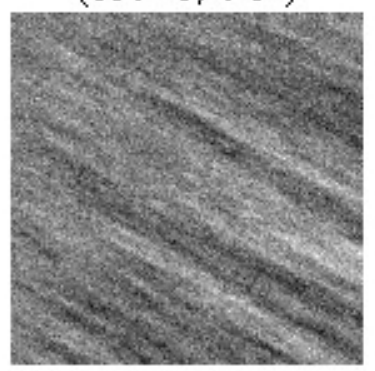

WSS_6

(850.86; 6.91)

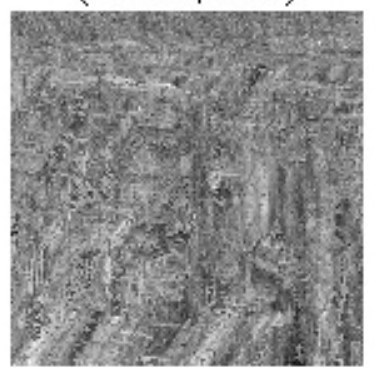

Loc WSS 2

(948.81; 6.99)

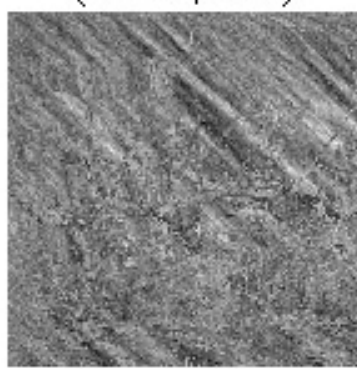

Loc_WSS_6

$(849.19 ; 6 . \overline{91})$

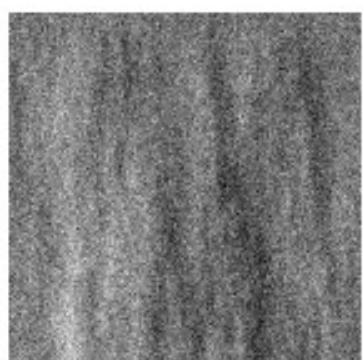

WSS_3

(767.72; 6.84)

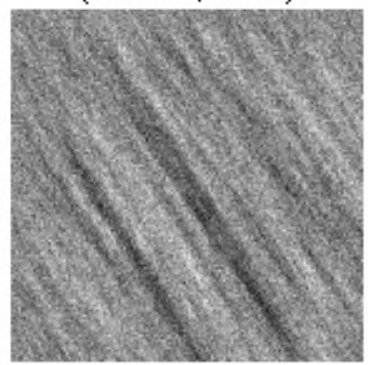

WSS 7

$(759.35 ; 6.82)$

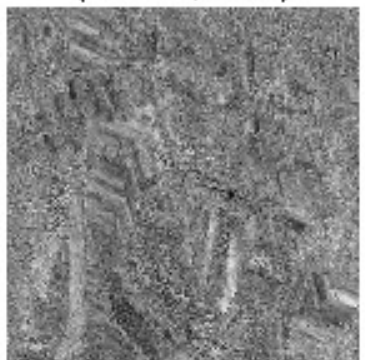

Loc WSS 3

$(889.59 ; 6 . \overline{94})$

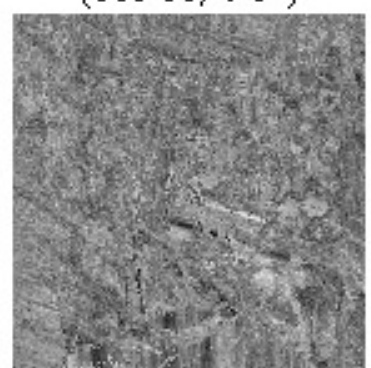

Loc_WSS_?

$(763.39 ; 6 . \overline{83})$

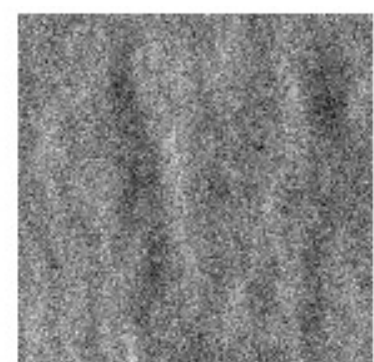

WSS 4

$(790.09 ; 6.86)$

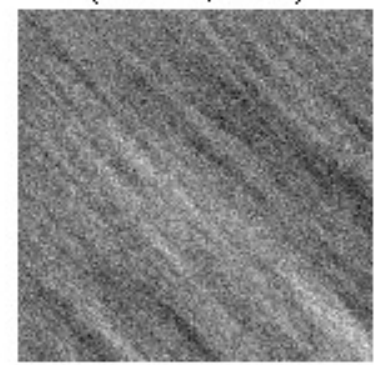

WSS 8

$(708.34 ; 6.78)$

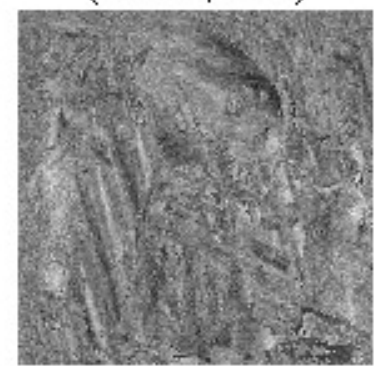

Loc WSS 4

$(811.18 ; 6 . \overline{8} 8)$

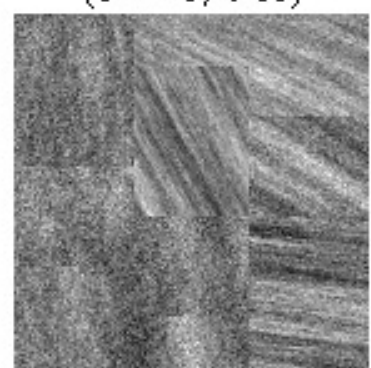

Loc_WSS_8

(973.22; 7.01)

Figure 6: Synthetic images, globally stationary (top first two rows) and locally stationary (last two rows). In the first row the causality is NSHP and QP in the second row. In the last two rows, the causality is either QP or NSHP per area (this choice has been made at random). The values indicated under each image correspond respectively to its variance and its first order entropy. 


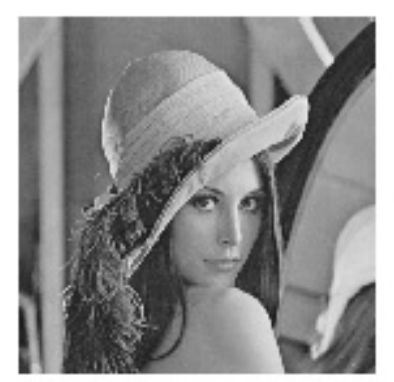

Lena

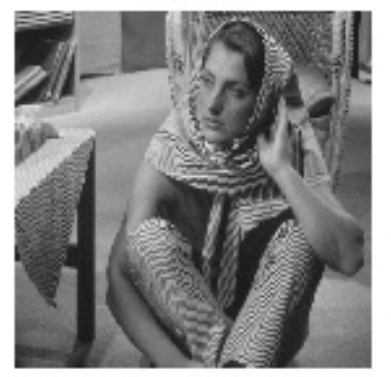

Barbora

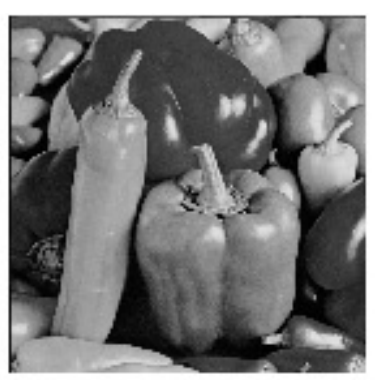

Peppers

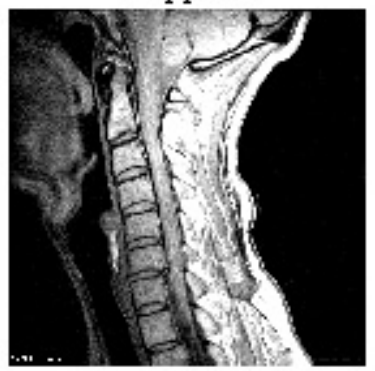

$M R I 1$

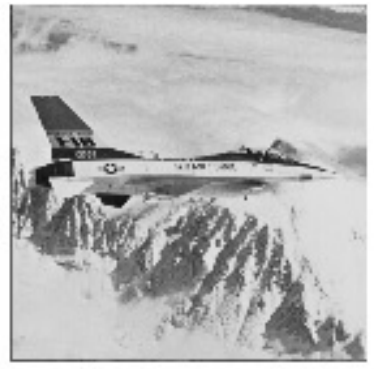

F16

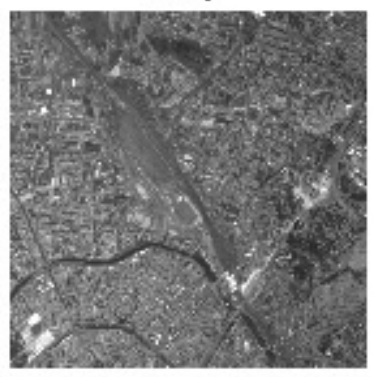

Satellitel

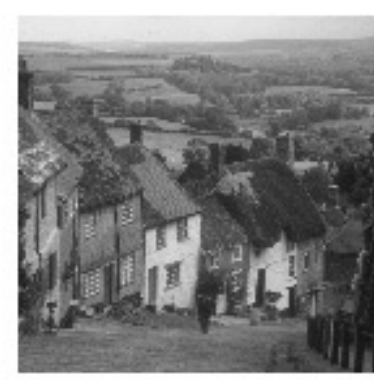

Goldkal

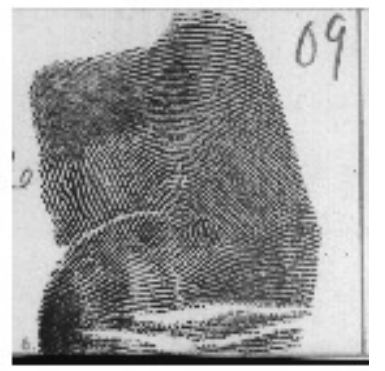

Finger 1

Figure 7: Some images used in our tests.

\begin{tabular}{|c||c|c||c|c||c|c||c|c||c|c||}
\hline \multicolumn{1}{|l||}{} & \multicolumn{2}{c||}{ GAE } & \multicolumn{2}{c||}{ LAE } & \multicolumn{2}{c||}{ Gerek et al. } & \multicolumn{2}{c||}{$(9,7)$} & \multicolumn{2}{c||}{$(5,3)$} \\
\hline & var. & ent. & var. & ent. & var. & ent. & var. & ent. & var. & ent. \\
\hline \hline WSS_1 & 108.1 & 5.42 & 112.8 & 5.44 & 185.1 & 5.81 & 216.5 & 5.93 & 325.7 & 6.22 \\
\hline WSS_2 & 181.1 & 5.80 & 185.7 & 5.81 & 231.7 & 6.21 & 359.2 & 6.29 & 548.1 & 6.59 \\
\hline WSS_3 & 189.5 & 5.83 & 195.4 & 5.85 & 324.7 & 6.20 & 355.7 & 6.28 & 542.1 & 6.58 \\
\hline WSS_4 & 278.4 & 6.11 & 287.4 & 6.13 & 509.7 & 6.54 & 555.5 & 6.60 & 849.3 & 6.91 \\
\hline WSS_5 & 143.4 & 5.63 & 146.8 & 5.64 & 271.4 & 6.08 & 269.6 & 6.08 & 404.8 & 6.38 \\
\hline WSS_6 & 176.5 & 5.78 & 189.1 & 5.80 & 315.6 & 6.19 & 364.6 & 6.30 & 556.4 & 6.61 \\
\hline WSS_7 & 172.1 & 5.76 & 176.3 & 5.78 & 304.7 & 6.17 & 366.8 & 6.31 & 559.3 & 6.61 \\
\hline WSS_8 & 194.8 & 5.85 & 199.6 & 5.87 & 353.7 & 6.27 & 401.7 & 6.37 & 614.0 & 6.68 \\
\hline \hline average & & 5.77 & & 5.79 & & 6.18 & & 6.27 & & 6.57 \\
\hline
\end{tabular}

Table 2: Variance (var.) and first order entropy (ent.) of the subband signal $x_{h}$ for different lifting scheme decompositions. The input signals WSS_ 1 to WSS_8 are described in $\S 4.1$.

\begin{tabular}{|c|c|c|c|c|c|c|c|c|c|c|}
\hline & \multicolumn{2}{|c|}{ GAE } & \multicolumn{2}{|c|}{ LAE } & \multicolumn{2}{|c|}{ Gerek et al. } & \multicolumn{2}{|c|}{$(9,7)$} & \multicolumn{2}{|c|}{$(5,3)$} \\
\hline & var. & ent. & var. & ent. & var. & ent. & var. & ent. & var. & ent. \\
\hline Loc_WSS_1 & 129.0 & 5.55 & $\overline{127.4}$ & 5.54 & 337.8 & 6.23 & 418.8 & 6.40 & 640.0 & 6.70 \\
\hline Loc WSS 2 & 124.0 & 5.51 & 117.8 & 5.46 & 344.5 & 6.22 & 409.9 & 6.36 & 631.3 & 6.68 \\
\hline Loc_WSS_3 & 141.4 & 5.61 & 141.6 & 5.60 & 356.8 & 6.27 & 471.3 & 6.47 & 719.1 & 6.77 \\
\hline Loc WSS 4 & 108.6 & 5.42 & 103.0 & 5.38 & 291.5 & 6.13 & 351.1 & 6.26 & 537.7 & 6.56 \\
\hline Loc_WSS_5 & 79.4 & 5.14 & 77.6 & 5.12 & 236.3 & 5.89 & 248.1 & 5.97 & 380.7 & 6.28 \\
\hline Loc WSS 6 & 115.5 & 5.46 & 116.5 & 5.44 & 310.7 & 6.17 & 369.7 & 6.29 & 565.6 & 6.60 \\
\hline Loc WSS 7 & 112.5 & 5.44 & 110.1 & 5.43 & 303.6 & 6.15 & 357.1 & 6.27 & 546.0 & 6.58 \\
\hline Loc_WSS_8 & 105.9 & 5.41 & 104.1 & 5.39 & 263.1 & 6.06 & 379.5 & 6.32 & 581.5 & 6.63 \\
\hline average & & 5.44 & & 5.42 & & 6.14 & & 6.29 & & 6.60 \\
\hline
\end{tabular}

Table 3: Variance (var.) and first order entropy (ent.) of the subband signal $x_{h}$ for different lifting scheme decompositions. The input signals Loc_WSS_ 1 to Loc_WSS_8 are described in $\S$ 4.1. 


\begin{tabular}{|c||c|c|c|c|c||c|c|}
\hline & $(2,2)$ & $(4,2)$ & $(4,4)$ & $(5,3)$ & S+P & GAE & LAE \\
\hline \hline Lena & 4.35 & $\mathbf{4 . 3 0}$ & $\mathbf{4 . 3 0}$ & 4.34 & 4.39 & 4.33 & 4.33 \\
\hline Goldhill & 4.84 & 4.83 & 4.83 & 4.84 & 4.95 & 4.81 & $\mathbf{4 . 8 0}$ \\
\hline Barbara & 4.99 & 4.86 & 4.82 & 4.99 & 4.83 & 4.69 & $\mathbf{4 . 6 8}$ \\
\hline F16 & 4.18 & $\mathbf{4 . 1 4}$ & $\mathbf{4 . 1 4}$ & 4.18 & 4.20 & 4.18 & 4.22 \\
\hline Mandrill & 6.11 & 6.09 & 6.08 & 6.11 & 6.15 & $\mathbf{6 . 0 7}$ & $\mathbf{6 . 0 7}$ \\
\hline Peppers & $\mathbf{4 . 5 8}$ & $\mathbf{4 . 5 8}$ & $\mathbf{4 . 5 8}$ & $\mathbf{4 . 5 8}$ & 4.70 & $\mathbf{4 . 5 8}$ & 4.67 \\
\hline IRM1 & $\mathbf{2 . 3 1}$ & 3.05 & 3.04 & 2.18 & 3.39 & 3.03 & 3.10 \\
\hline IRM2 & 2.35 & 3.10 & 3.10 & $\mathbf{2 . 2 3}$ & 3.49 & 2.78 & 3.05 \\
\hline IRM3 & 5.18 & 5.11 & 5.11 & 5.18 & 5.06 & $\mathbf{4 . 8 8}$ & 5.01 \\
\hline IRM4 & 4.54 & 4.47 & 4.48 & 4.52 & $\mathbf{4 . 3 6}$ & 4.43 & 4.38 \\
\hline IRM5 & 4.24 & 4.11 & 4.12 & 4.24 & 4.04 & 4.35 & $\mathbf{4 . 0 3}$ \\
\hline IRM6 & 1.84 & 2.36 & 2.36 & $\mathbf{1 . 7 4}$ & 2.60 & 3.47 & 2.39 \\
\hline Pentagone & 5.27 & 5.27 & $\mathbf{5 . 2 6}$ & 5.27 & 5.72 & 5.27 & 5.28 \\
\hline Sanfrancisco & 4.88 & 4.86 & 4.85 & 4.88 & 5.35 & $\mathbf{4 . 8 5}$ & $\mathbf{4 . 8 5}$ \\
\hline Okland & 4.38 & 4.37 & 4.36 & 4.38 & 4.84 & $\mathbf{4 . 3 5}$ & 4.38 \\
\hline Toulouse & 5.23 & 5.15 & 5.15 & 5.23 & 5.92 & $\mathbf{5 . 0 5}$ & 5.11 \\
\hline Genes & 4.28 & 4.25 & 4.25 & 4.28 & 5.01 & $\mathbf{4 . 2 1}$ & 4.24 \\
\hline Airplane* & 4.51 & 4.52 & 4.51 & 4.51 & 4.80 & $\mathbf{4 . 4 6}$ & 4.50 \\
\hline Airport* & 5.40 & 5.38 & 5.38 & 5.40 & 5.82 & $\mathbf{5 . 2 1}$ & 5.31 \\
\hline Finger1 & 4.85 & 4.61 & 4.62 & 4.85 & 4.53 & $\mathbf{4 . 3 3}$ & 4.40 \\
\hline Finger2 & 4.25 & 4.09 & 4.10 & 4.25 & 4.09 & $\mathbf{3 . 8 9}$ & 3.94 \\
\hline Finger3 & 4.78 & 4.56 & 4.60 & 4.78 & 4.54 & $\mathbf{4 . 3 6}$ & 4.40 \\
\hline
\end{tabular}

Table 4: Average of the first order entropies of the image decomposed with 5 or 6 (images with an asterisk) levels of decomposition. For the GAE method, the values of the parameters are $(p, q)=(3,3)$ and a separable decimation. For the LAE method, the values of the parameters area separable decimation, $\alpha=0.9995$, $\left(r_{1}, r_{2}\right)=(6,2)$ for MRI medical images and $\left(r_{1}, r_{2}\right)=(8,4)$ for all other image families. 


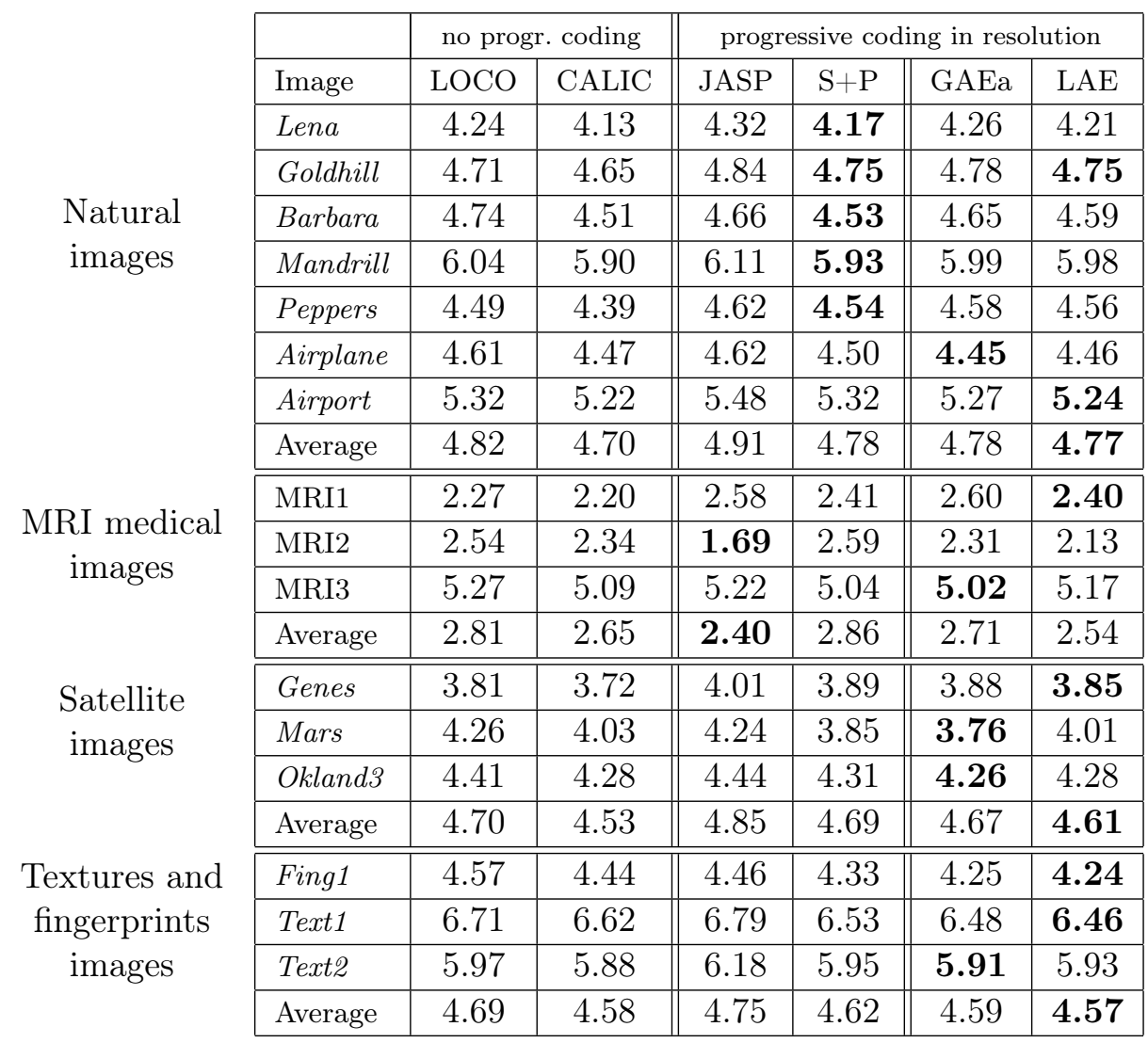

Table 5: Bit-rates (in bpp) for the codecs S+P, LOCO (i.e., JPEG-LS), CALIC, Jasper (i.e., JPEG2000) and the proposed methods. Only the four coders GAEa, LAE, Jasper and $S+P$ allow for a progressive coding in resolution. The rows "average" are computed for each family of images.

\begin{tabular}{|c|c|c|c|c||c|c|}
\hline time & LOCO & CALIC & JASP & S+P & GAEa & LAE \\
\hline coding & 0.12 & 0.21 & 0.40 & 0.36 & 3.19 & 3.27 \\
\hline decoding & 0.11 & 0.26 & 0.37 & 0.35 & 0.46 & 3.20 \\
\hline
\end{tabular}

Table 6: Mean coding and mean decoding time on images of dimension $512 \times 512$ and coded on 8 bpp, expressed in seconds. The codecs have been implemented on a PC PIII $700 \mathrm{MHz}$, with $256 \mathrm{Mo}$ of RAM. 